C-A/AP/\#422 Jan. 2011

\title{
Energy Upgrade as Regards Quench Performance
}

\author{
W.W. MacKay, S. Tepikian
}

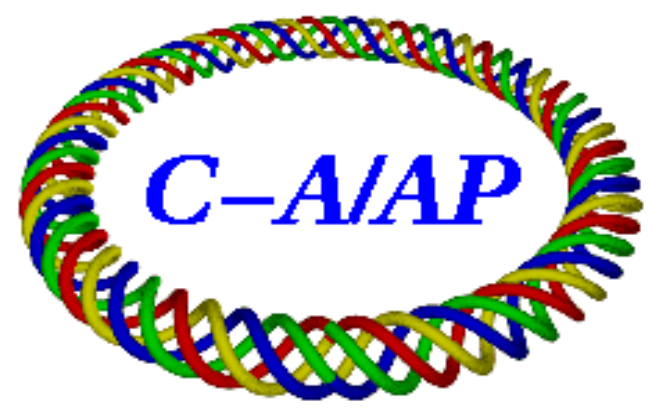

\section{Collider-Accelerator Department Brookhaven National Laboratory Upton, NY 11973}

Notice: This document has been authorized by employees of Brookhaven Science Associates, LLC under Contract No. DE-AC02-98CH10886 with the 
30 January, 2011

\title{
Energy upgrade as regards quench performance
}

\author{
W. W. MacKay, and S. Tepikian
}

\begin{abstract}
Since the cross section for $\mathrm{W}$ production increases rapidly with energy, we consider the possibility of increasing the collision energy of polarized protons at RHIC. The limits of present hardware are examined with a particular emphasis on the quench training performance of magnets. Ignoring the limits of the DX magnets, the short-sample currents for the main arc $(8 \mathrm{~cm})$ dipoles could allow an increase of more than $30 \%$, however we estimate 400 to 500 training quenches for the just $8 \mathrm{~cm}$ dipoles to reach this level. We propose that a $10 \%$ increase in energy might be achieved with the present hardware configuration. Raising the beam energy to $275 \mathrm{GeV}(\sqrt{s}=550 \mathrm{GeV})$ should increase the $\mathrm{W}$ production rate by almost $50 \%$ from the $250 \mathrm{GeV}$ level for the same optics with identical $\beta^{*}$ 's at the collision points.
\end{abstract}

\section{Introduction}

One of the prominent reasons for considering an energy upgrade for RHIC is the potential increase in W-boson production from collisions of polarized proton beams. Werner Vogelsang provided[1] the data for Fig. 1 which illustrates the relative dependence of $\mathrm{W}^{+}$production from $\mathrm{p}+\mathrm{p}$ collisions as a function of beam energy. Above $\sqrt{s} \sim 600 \mathrm{GeV}$ the cross section scales linearly with $s$. For an increase of beam energy from 250 to $325 \mathrm{GeV}$, one could expect about a twofold increase in the production rate of W's[1,2,3].

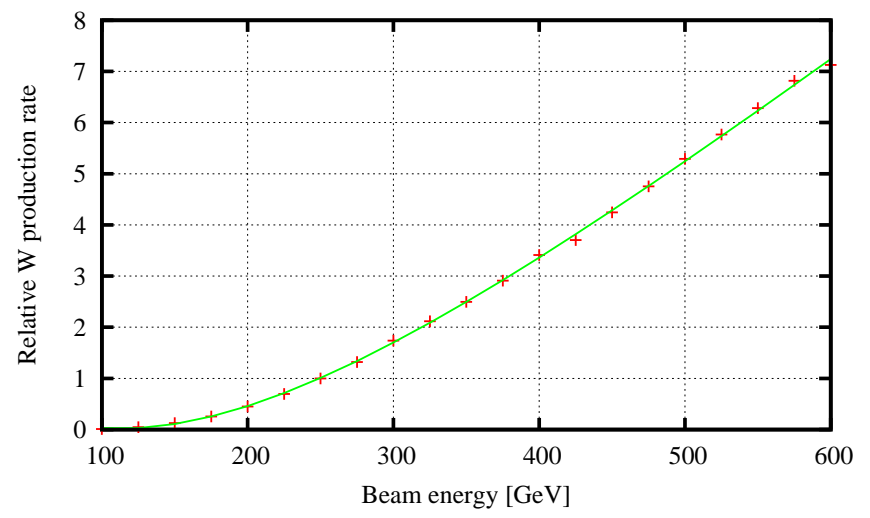

Figure 1. Relative cross section for $\mathrm{p}+\mathrm{p} \rightarrow \mathrm{W}^{+}+\mathrm{X}$ as a function of beam energy $(U=\sqrt{s} / 2)$ normalized to the cross section $\sigma_{500}$ at a beam energy of $250 \mathrm{GeV}$. The green curve is a fit to Vogelsang's points (red pluses) using the formula $W(U)=a_{0}+a_{1} U+a_{2} e^{a_{3} U}$, with $a_{0}=-5.61944 \pm 0.45159$, $a_{1}=0.02121 \pm 0.00069, a_{2}=6.76792 \pm 0.25169$, and $a_{3}=-0.00652 \pm 0.00078$. This is not a good fit for energies below $\sim 100 \mathrm{GeV}$; however for the purposes of this paper, we are really only interested in the scaling from 250 to $350 \mathrm{GeV}$

While the short-sample currents for the superconducting magnets are high, the performance of the actual magnets is somewhat lower. In particular, some of the DX dipole magnets require frequent retraining after thermal cycling for operation at the design rigidity of $833 \mathrm{Tm}$ ( $250 \mathrm{GeV}$ protons). In addition, we can expect that the D0 dipole and triplet quadrupole magnets may require retraining at higher operating currents. While a few triplet quadrupoles were tested to currents in excess of $8000 \mathrm{~A}$ (up to $9000 \mathrm{~A}$ for QRI102), it was thought that magnetic forces exceeded the mechanical strength of the collar preloading, since quadrupoles trained up $8000 \mathrm{~A}$ then had subsequent quenches at considerably lower currents.

Allowing nonzero crossing angles at IP's can reduce the required currents of the DX and D0 dipoles at a given energy, however the peak luminosity will drop as the crossing angle is increased from zero (see Fig 2 left). Curves are shown for normalized $95 \%$ emittances $(\pi \epsilon)$ of $15 \pi$ and $40 \pi \mu \mathrm{m}$ as well as with and without a $\pm 10 \mathrm{~cm}$ vertex cut. Since a crossing angle reduces the longitudinal extent of the collision diamond (Fig. 2 right) the relative luminosity will fall off more slowly with a longitudinal cut of $\pm 10 \mathrm{~cm}$. 

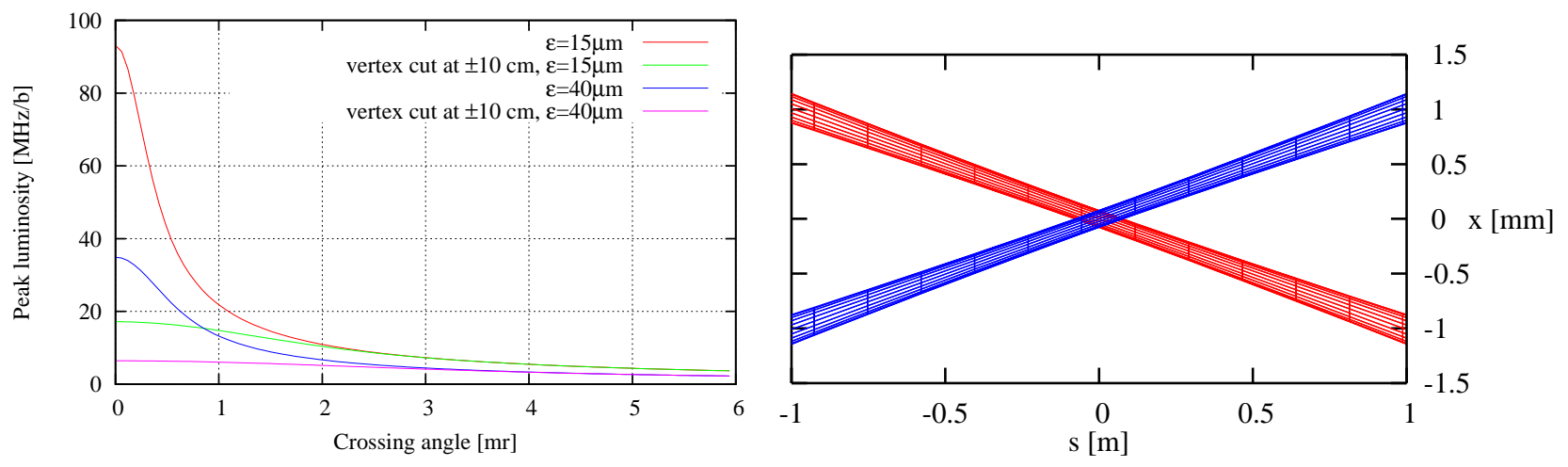

Figure 2. Left: Peak luminosity at one IP versus total crossing angle at $325 \mathrm{GeV}$ under the assumption identical conditions for both beams: 109 colliding bunches per turn with $1 \times 10^{11}$ protons per bunch, $\beta_{x}^{*}=\beta_{y}^{*}=0.7 \mathrm{~m}, \sigma_{z}=0.87 \mathrm{~m}$, and $\sigma_{p} / p=7 \times 10^{-4}$. Right: Simulation of the crossing diamond for $1 \sigma$ beam envelopes ( $15 \pi$ emittances) at $278 \mathrm{GeV}$ with a crossing angle of $2 \mathrm{mrad}$. This is the crossing angle one would obtain at $278 \mathrm{GeV}$ if the DX and D0 magnets were operated with currents for a $0^{\circ}$ crossing angle at $250 \mathrm{GeV}$.
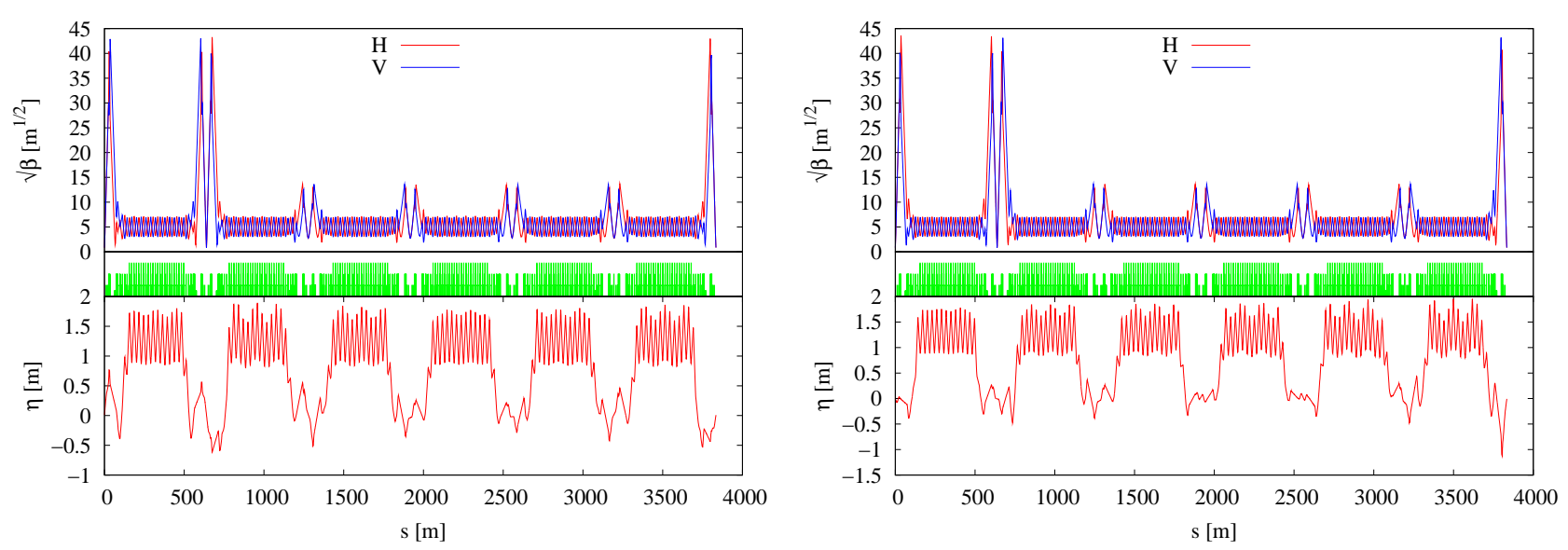

Figure 3. Transverse envelope (top) and dispersion (bottom) functions of beam versus azimuthal positions around the rings. Functions are plotted for the March $2009250 \mathrm{GeV}$ lattice. Left: Blue clockwise ring, Right: Yellow counter-clockwise ring.

In order to study the limitations of an energy upgrade from quench performance, current limits of power supplies and cryogenic feedthroughs, we have taken ramp data from an actual RHIC ramp to $250 \mathrm{GeV}$ with polarized protons. The fill was \#10348 from 12 March, 2009 with $\beta^{*}$ s of $0.7 \mathrm{~m}$ at STAR and PHENIX and $7 \mathrm{~m}$ at the other four IR crossing points. Fig. 3 shows the corresponding model optical functions for the Blue and Yellow rings.

\section{D0 and DX apertures and crossing angles}

Fig. 4 shows the physical aperture limits for the Blue ring from the center of STAR to beyond the Q4 quadrupole on the 6 o'clock side of STAR. The 5 o'clock side is the mirror image, and the Yellow ring apertures are the same as the Blue ring. The PHENIX apertures are identical to the STAR apertures in the regions from the DX dipoles to the Q4 quadrupoles. 


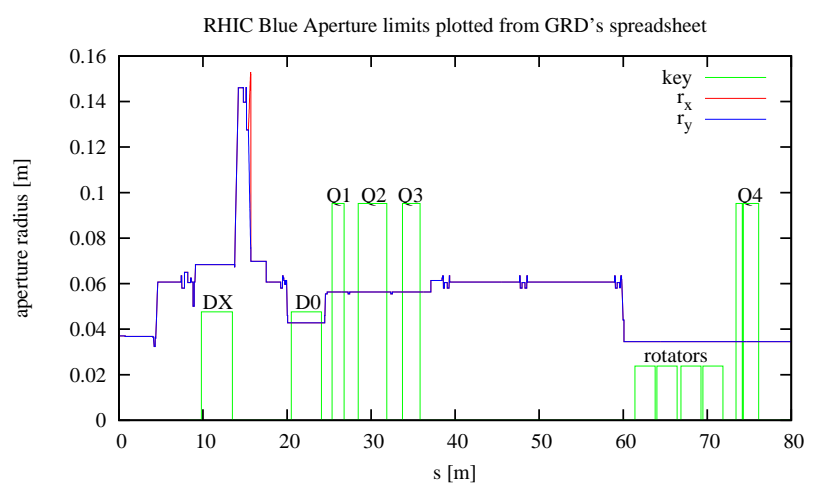

Figure 4. Aperture limits of beam pipe near the interaction region. This plot shows the aperture for the 6 o'clock side of STAR in the Blue ring. The apertures in the DX magnets through the triplets are essentially identical in both rings at all 6 IR's.

Assuming "round" beams at the IP, the beam envelope at the interaction region may be parameterized in the transverse dimensions as

$$
\sigma_{x}(s, \gamma)=\sqrt{\frac{\epsilon_{95 \%}^{N}}{6 \beta \gamma} \beta_{x}(s)+\left(\eta_{x}(s) \frac{\sigma_{p}}{p}\right)^{2}}, \quad \text { and } \quad \sigma_{y}(s, \gamma)=\sqrt{\frac{\epsilon_{95 \%}^{N}}{6 \beta \gamma} \beta_{y}(s)},
$$

where $s$ is measured from the IP and

$$
\beta_{x, y}(s)=\beta^{*}+\frac{s^{2}}{\beta^{*}} .
$$

The horizontal dispersion $\eta_{x}^{*} \simeq 0$ at the IP is quite small, so the effect of dispersion on the horizontal beam size is practically negligible. Here $\gamma$ is the usual Lorentz factor of the beam, and we assume a Gaussian bunch shape with transverse normalized $95 \%$ emittances $\left(\pi \epsilon_{95 \%}^{N}\right)$ and $\sigma_{z}=0.87 \mathrm{~m}$ in the longitudinal direction. Luminosity calculations use the algorithms described in Ref. [4].

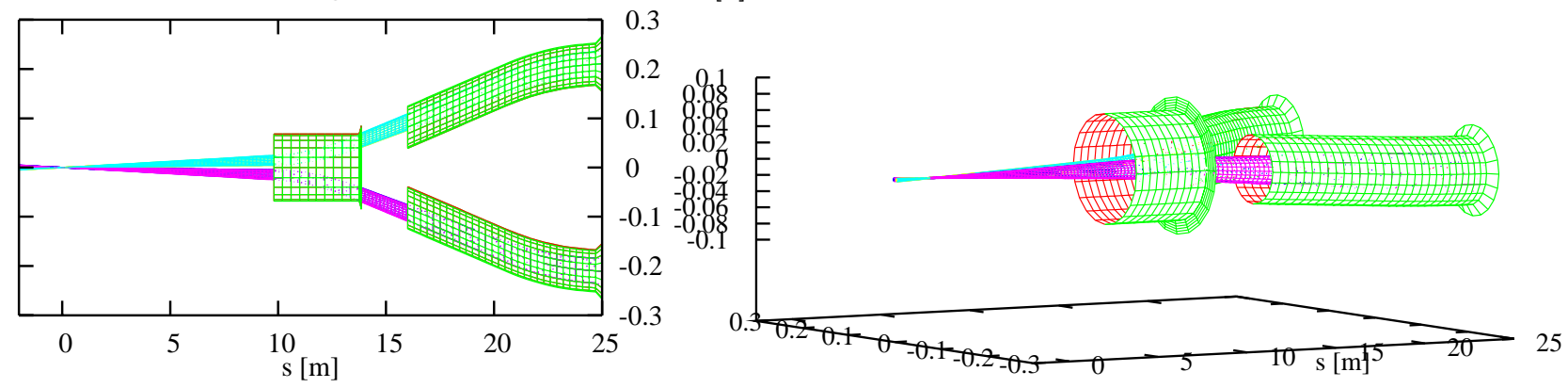

Figure 5. DX and D0 pipe apertures with a $\pm 6 \sigma$ beam envelope. The $6 \sigma$ beam envelopes are shown for a $292 \mathrm{GeV}$ beam with $\beta^{*} \mathrm{~m}$, transverse emittance $\pi \epsilon=40 \pi \mu \mathrm{m}$ and a $3.02 \mathrm{mr}$ total crossing angle. Left: top view, Right: side view.

For a 2 mrad crossing angle, we must change the strengths of the D0 and DX magnets at a given IR to make a $+1 \mathrm{mrad}$ orbit shift in the closed orbit of one beam, and a $-1 \mathrm{mrad}$ orbit shift in the closed orbit of the other beam. This is most easily done by lowering the fields of D0 and DX magnets. In order to make these local angle bumps with the closed orbits being unperturbed in the rest of the rings, the DX and D0 fields should be scaled according to

$$
\left(\frac{\Delta B}{B_{0}}\right)_{\mathrm{DX}}=-0.055505, \text { and }\left(\frac{\Delta B}{B_{0}}\right)_{\mathrm{D} 0}=-0.0360095
$$

respectively per milliradian of desired crossing angle.

Fig. 5 shows the physical aperture in the DX and D0 magnets with an envelope corresponding to $\pm 6 \sigma$ of a $40 \pi \mu \mathrm{m}$ emittance beam at $292 \mathrm{GeV}$ and a $3 \mathrm{mrad}$ crossing angle. Here we have again assumed $\beta^{*}=0.7 \mathrm{~m}$. 


\section{Main dipole and quadrupole magnets}

Table 1 lists the types and total numbers of main dipole and quadrupole magnets in both rings of RHIC. we have grouped the magnets with identical transverse cross sections together (e. g. $8 \mathrm{~cm}$ dipoles of different lengths) since the quench performance is assumed not to depend on the length of a magnet.

Table 1. Numbers of main dipoles and quadrupoles in RHIC.

\begin{tabular}{lllrr} 
Magnet type & Style & \multicolumn{1}{c}{ Serial number prefix } & Coil ID & $N_{\text {tot }}$ \\
\hline Dipole & DRG & DRG, DR8, D5I, D5O, D96 & $8[\mathrm{~cm}]$ & 360 \\
Dipole & D0 & DRZ & $10[\mathrm{~cm}]$ & 24 \\
Dipole & DX & DRX & $18[\mathrm{~cm}]$ & 12 \\
Quadrupole & QRG & QRG, QR4, QR7 & $8[\mathrm{~cm}]$ & 420 \\
Quadrupole & QRI & QRI, QRJ, QRK & $13[\mathrm{~cm}]$ & 72
\end{tabular}

\subsection{Quadrupole magnets}

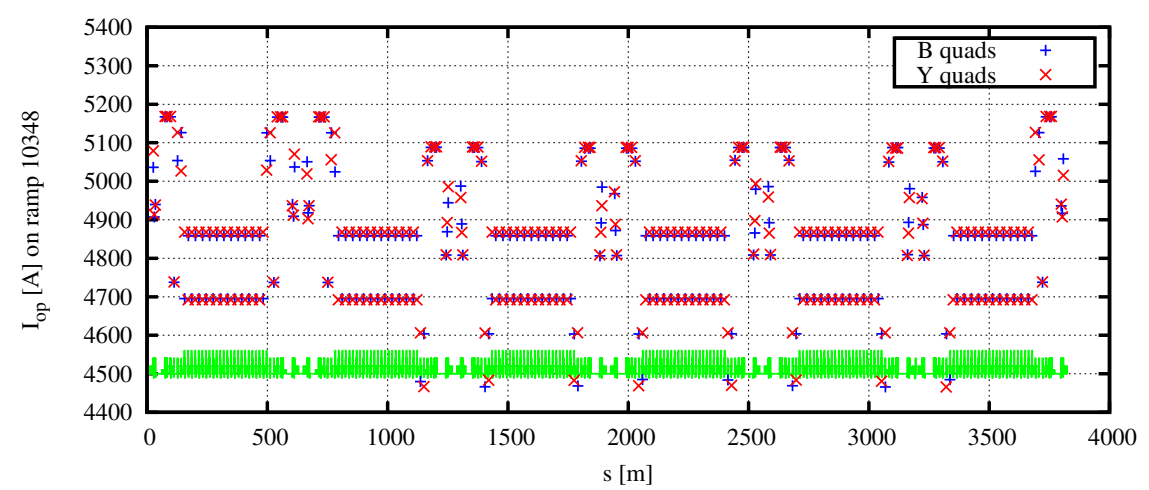

Figure 6. Maximum currents in quadrupoles for the ramp of Fill 10348.

Using the ramp from Fill 10348, Fig. 6 shows the peak operating current of the quadrupoles versus longitudinal position around the ring for both rings. Estimates of the quench probabilities of arc and triplet quadrupoles are given in the next two subsections.

\subsubsection{Estimation of quench probabilities for the 8-cm QRG quadrupoles}

There are a total of $N_{\text {tot }}=420$ arc quadrupoles (style QRG with $8 \mathrm{~cm}$ coil ID) installed in both rings, of which $N_{\text {sample }}=95$ were cold tested and trained at the Magnet Facility up to $\sim 8000$ A. From Fill 10348 (see Fig. 6), the maximum current for any QRG quadrupole was 5168 A (for Q4-Q6 of IR's 6 and 8).

There were 19 other QRG quads which were tested only in a horizontal dewar, but the quench plateaus of this sample were lower than for QRG's tested in vertical dewars. These lower plateaus were ascribed to the presence of a warm bore tube which caused a larger heat load in the horizontal dewar. Since the QRG magnets in RHIC operate without a warm bore tube, we did not include these magnets in the statistical sample.

The probability for a given magnet to have training quenches below a given current, $I$ is modeled as the sum of the quenches less than $I$ from the sample divided by $N_{\text {sample: }}$ :

$$
P(I)=\frac{\# \text { of quenches below } I}{N_{\text {sample }}}
$$

The data were fit to an exponential for currents from 6200 to $7250 \mathrm{~A}$ as shown in Fig. 7. We used gnuplot [9] to fit and plot the data. 

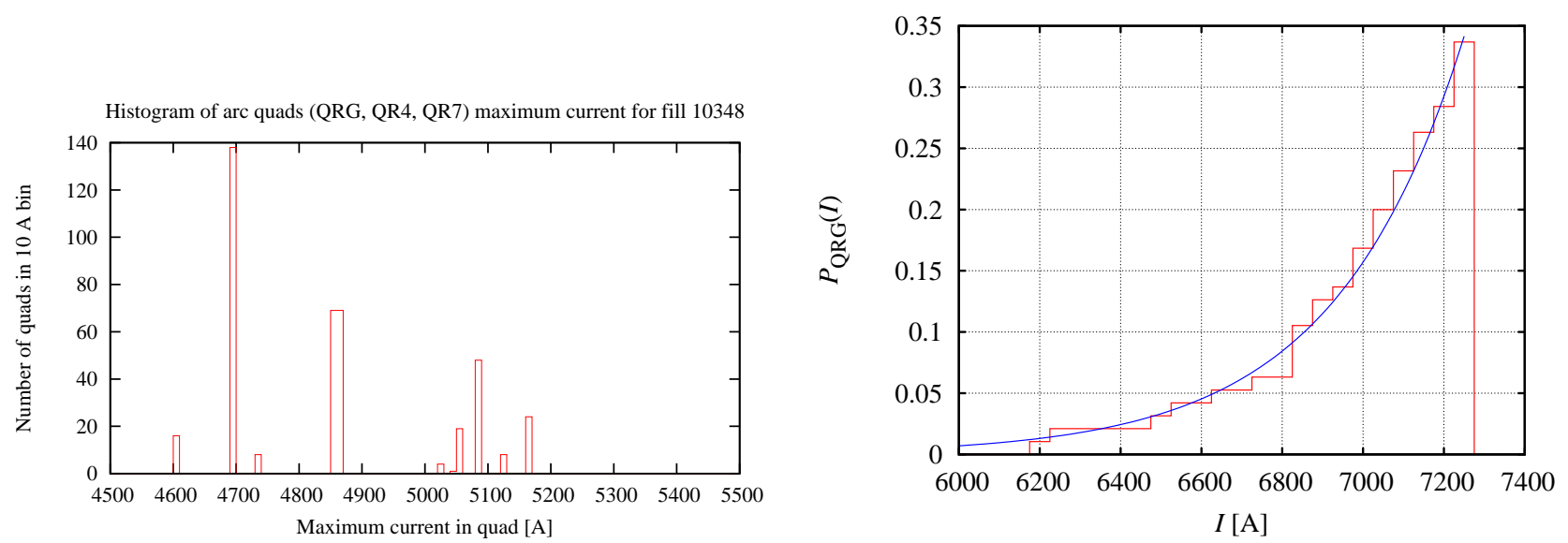

Figure 7. Left: Histogram of the $250 \mathrm{GeV}$ peak operating currents $I_{\max }$ for the $8 \mathrm{~cm}$ style (QRG) arc quads (including QRG, QR4, and QR7 quads). RHIC has a total of 420 QRG quads. Right: Estimation of training quench probability $P(I)=\exp (-23.74+0.003005 I)$ for a single QRG quad to be operated at current $I$. The data in the red histogram were fit to an exponential (blue curve).

Since the magnets were starting to saturate at these energies, a cubic polynomial was fit to the measured transfer function $T(I)$ for two QRG magnets in the region from 4 to $7.5 \mathrm{kA}$ (see Fig. 8). The estimated number of quenches was then calculated by the formula

$$
N_{\text {est }}(r)=\frac{N_{\text {tot }}}{N_{\text {sample }}} \sum_{j=1}^{N_{\mathrm{sample}}} P\left(r I_{\mathrm{op}, j} \frac{T\left(I_{\mathrm{op}, n}\right)}{T\left(r I_{\mathrm{op}, j}\right)}\right),
$$

where

$$
r=\frac{U}{250 \mathrm{GeV}}
$$

is the ratio of the desired beam energy $U$ to the design operating energy of $250 \mathrm{GeV}$. Here we have applied the ratio $T\left(I_{\mathrm{op}, n}\right) / T\left(r I_{\mathrm{op}, j}\right)$ as a first order correction to the current to account for saturation of the iron.
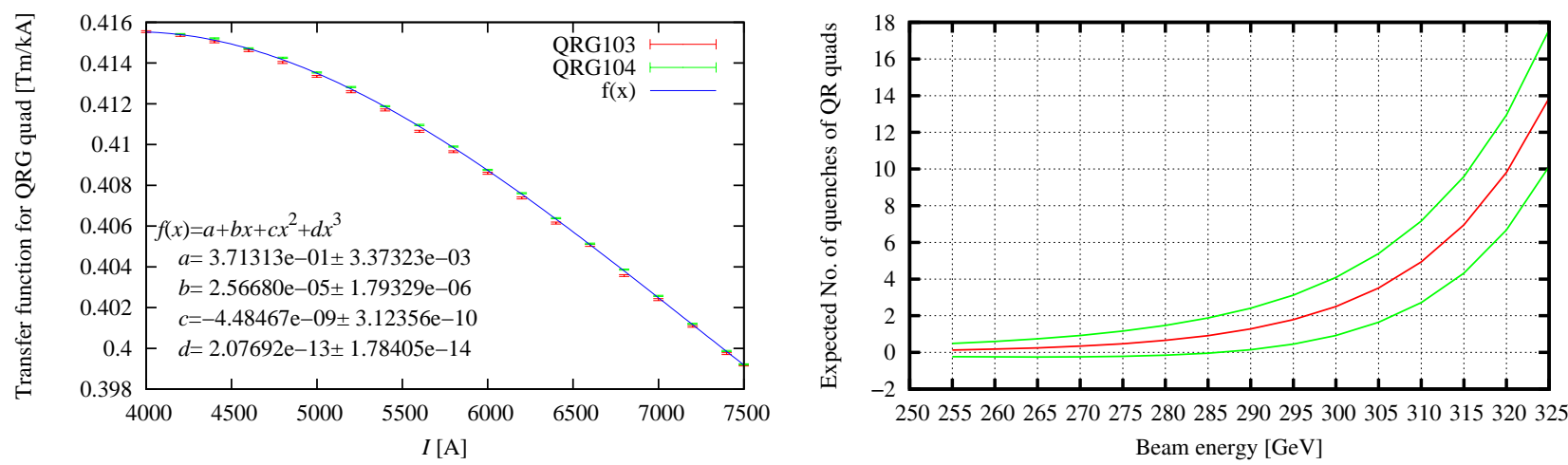

Figure 8. Left: Fit of a cubic polynomial to measured transfer-function data for two QRG quadrupoles. Right: Expected number of training quenches of the $8 \mathrm{~cm}$ QRG quads (red curve). The green curves bracket the $\pm 1 \sigma$ error bands.

The curves in Fig. 7 along with the saturation correction were combined to estimate the expected number of quenches of QRG quads as a function of beam energy beyond $250 \mathrm{GeV}$. The result is shown in Fig. 8. This estimate may be somewhat high, since about $1 / 4$ of the QRG quads were previously trained in the test facility; however, the magnets were subsequently moved into the tunnel and have had several thermal cycles over the last decade. If we were to assume that the previously trained QRG quads all remembered their training, then we could reduce the estimate of quenches by about $25 \%$, e. g. at $325 \mathrm{GeV}$ from $13.8 \pm 3.7$ quenches down to $10.4 \pm 3.2$ quenches. 


\subsection{2. $13 \mathrm{~cm}$ triplet (QRI) quadrupoles}
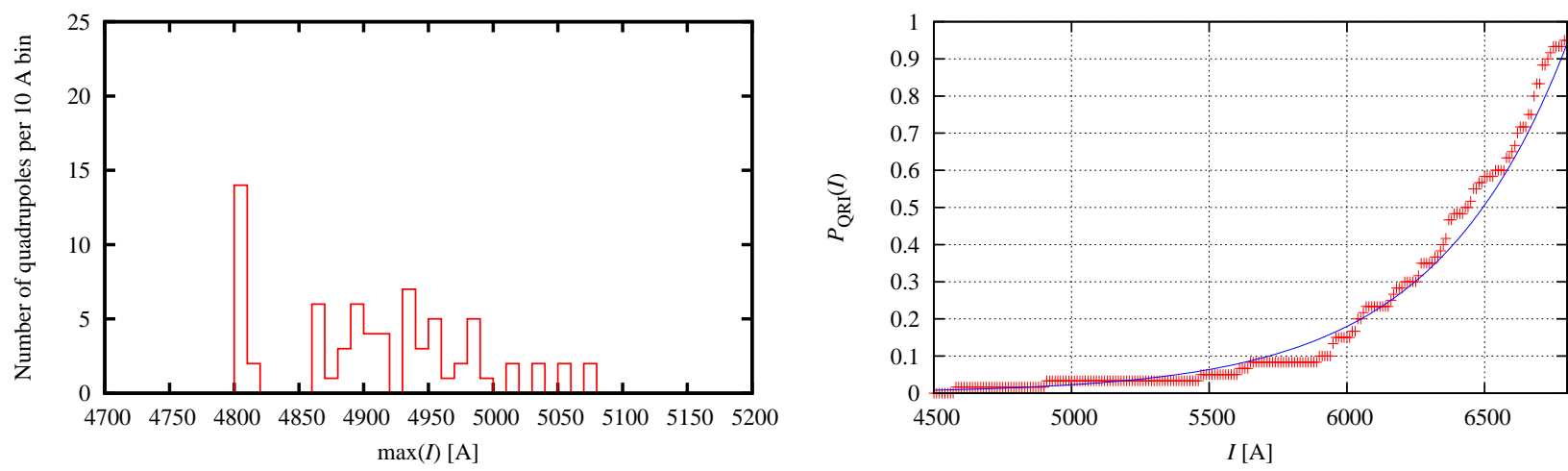

Figure 9. Left: Distribution of maximum currents for triplet quads on Ramp 10348. Right: Probability of a single magnet having a training quench vs current. The data was limited to currents below $6800 \mathrm{~A}$, since not all the magnets trained were trained to higher currents. Statistics for this probability function were compiled from training data for a sample of $N_{\text {sample }}=60$ magnets. The curve is a fit of the exponential function $f(I)=\exp \left(a_{0}+a_{1} I\right)$ to the data.
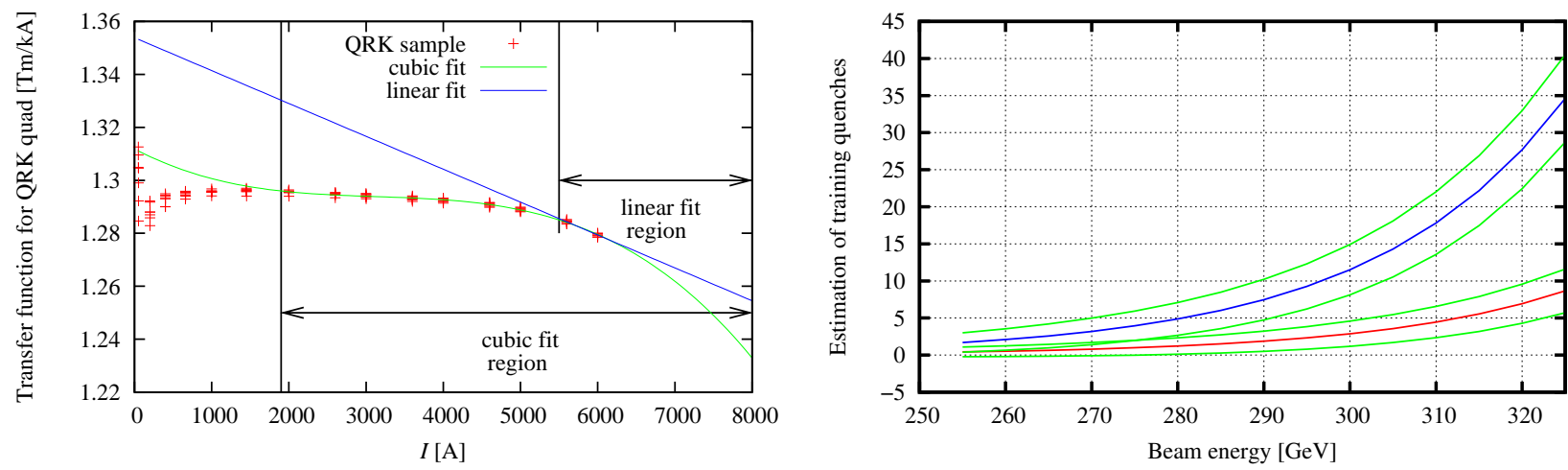

Figure 10. Left: Transfer function for QRI style magnets fit to data from a sample of the 7 magnets: QRK120, QRK122, QRK123, QRK124, QRK125, QRK126, and QRK127. The region above $1900 \mathrm{~A}$ was fit to the cubic polynomial $f(I)=a_{0}+a_{1} I+a_{2} I^{2}+a_{3} I^{3}$. The last two data clusters were also fit to a straight line for comparison; however the more pessimistic cubic function was used to correct currents for saturation. Right: Estimated number of triplet quad quenches vs energy. The blue curve indicates the estimated number of quenches assuming that none of the $N_{\text {tot }}=72$ installed magnets were to retain any previous training at the test facility. For the lower red curve, the estimate has been scaled by (72-54)/72 assuming a retention of training for the 54 pretrained magnets which were installed in RHIC. The green curves indicate the $\pm 1 \sigma$ envelopes about the red and blue curves.

Triplet quadrupoles which were trained up to 8000 A typically then had a quench at lower current which required retraining. Analysis of quench probabilities and estimated number of training for the triplet quadrupoles followed the same method as for the QRG quadrupoles. Figs. 9 and 10 show the results of this analysis. Two estimates for the expected number of training quenches are given in the final plot: one assuming that none of the QRI magnets "remember" any previous training from the magnet facility, and the other assuming that the 54 magnets in the ring which were previously trained to high currents would retain their training. Reality will be somewhere between the two estimates, but we expect the number to be close to the lower estimate.

\subsubsection{Trim quadrupoles: tq4, tq5, tq 6}

Eighteen QRT trim quadrupoles were cold tested in the magnet facility were performed. All achieved 199 A or slightly higher with either no quenches or only one training quench. For Fill 10348, the maximum operating currents were 99 A for the "tq4" magnets in the STAR and PHENIX IR's, so there should be plenty of margin 
without any training of any "tq" magnets required. The cryogenic feedthroughs for the QRT magnets are rated at $150 \mathrm{~A}$, so there is a $50 \%$ margin to increase their currents.

\subsection{Main dipole magnets}

\subsubsection{Main arc dipoles}

For the $N_{\text {tot }}=360$ DRG dipoles (prefixes: DRG, DR8, D5I, D5O, D96), the maximum required current for Ramp 10348 was $\max \left(I_{\mathrm{op}}\right)=5057 \mathrm{~A}$. With a $35 \%$ margin, this requirement increases to $6733 \mathrm{~A}$. Of the 53 DRG magnets which were trained up to a plateau in the test facility only two did not a plateau above the $35 \%$ margin.

It is worth noting a few remarks about three of the magnets which were cold tested and installed into RHIC:

- DRG102 (yo5-dh12): Did not reach a plateau before thermal cycling; the training seemed erratic. After a thermal cycle, the performance was improved and it did reach a plateau around $7100 \mathrm{~A}$.

- DRG108 (yo5-dh18): Plateaued at 6709 A; however it was suspected that there may have been a bad solder joint or conductor damage (in the leads?) for the test.

- DRG133 (yi6-dh18): Was noted to be erratic with plateaus of 6500 A before thermal cycling and $6400 \mathrm{~A}$ after thermal cycling.

At $325 \mathrm{GeV}$ the DRG operating operating current should be around

$$
5058 \times \frac{325}{250} \times \frac{6.4}{6.1} \simeq 6900 \mathrm{~A},
$$

where the 6.4/6.1 ratio was estimated from the transfer function plot in Fig. 11. For the $325 \mathrm{GeV}$ energy level, perhaps as many as

$$
\frac{360}{53} \times(2 \text { to } 3) \simeq 13 \text { to } 20
$$

magnets might need to be replaced. The statistics for such weak performers is really too poor to make any better estimate.

For the DRG dipoles, Figs. 11 and 12 show the results of the quench analysis similar to the main quadrupoles. Again the number of expected quenches in Fig. 12 assumes that none of the 62 dipoles retained their previous training. Assuming that the previous training was retained, the curves could be scaled down by $(360-62) / 360=0.828$ at $325 \mathrm{GeV}$ from around $501 \pm 22$ down to $415 \pm 20$ expected training quenches. This large number of quenches is too large to practically reach an energy of $325 \mathrm{GeV}$. A more reasonable maximum energy might be in the range of 275 to $290 \mathrm{GeV}$ ( $8.5 \pm 2.9$ to $28 \pm 5$ DRG training quenches).
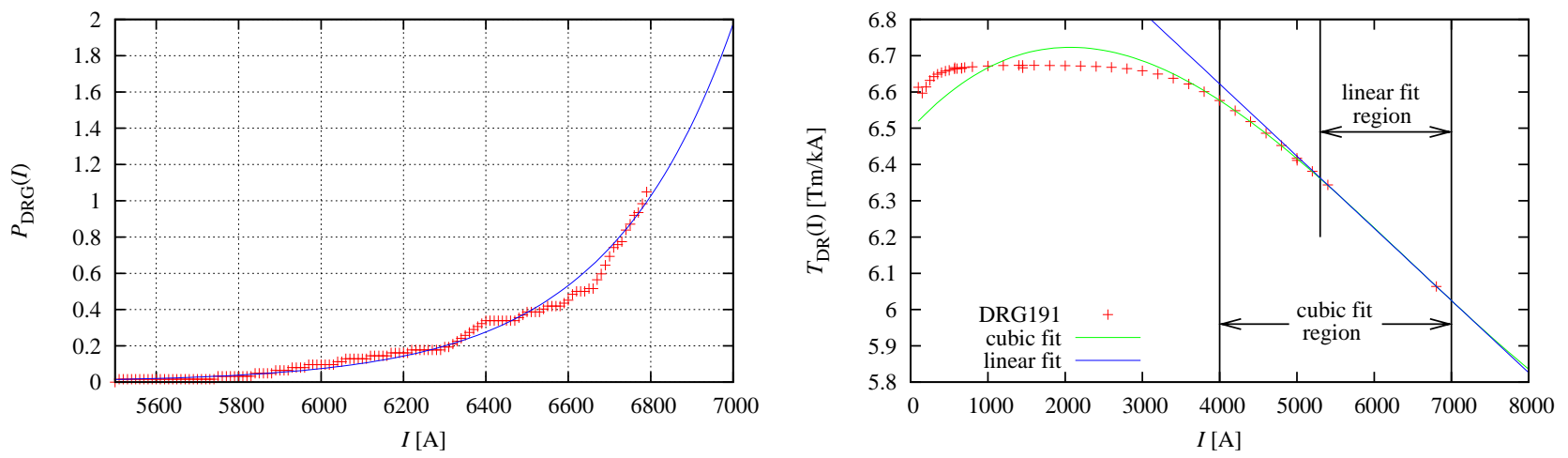

Figure 11. Left: Fit (blue curve) of quench probability for DRG dipoles. Red points are from training quenches of sample of $N_{\text {sample }}=62$ dipoles. The fit to $P_{\mathrm{DRG}}(I)=\exp (a+b I)$ is shown in blue. Right: Transfer function for DRG191. Points from 4000 to 7000 A were fit to a cubic polynomial. A line was fit to the last two points. These two functions were combined to produce an estimate of the transfer function above $4000 \mathrm{~A}$ by using the cubic polynomial between 4000 and $5300 \mathrm{~A}$ and the line above $5300 \mathrm{~A}$. 


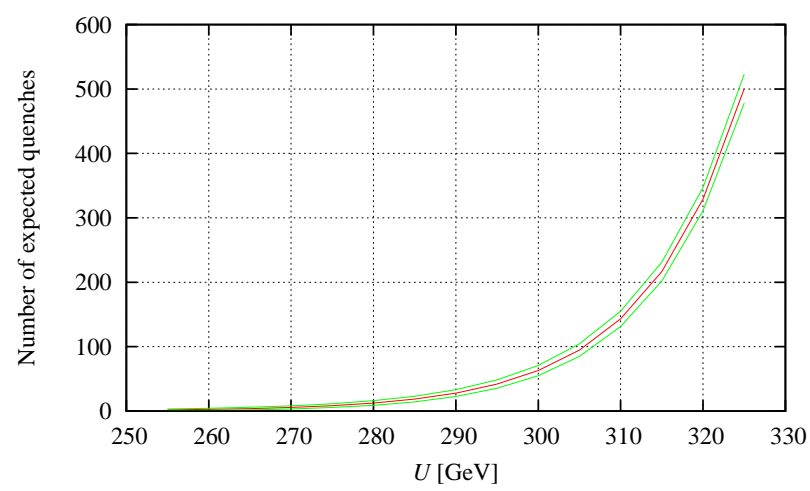

Figure 12. Estimated number of quenches of DRG magnets (red) with $\pm 1 \sigma$ bands (green).

\subsection{D0 dipoles}

For a zero crossing angle at $250 \mathrm{GeV}$, the operating current in the D0 magnets is $I_{250}=4988 \mathrm{~A}$. To estimate the quench probability we used $N_{\text {sample }}=19$ magnets which were cold tested at the magnet facility. While RHIC only requires $N_{\text {tot }}=24$ D0 magnets, 26 were built. Only the 24 of the magnets were cold tested at the magnet facility (see Fig. 13 with only 22 of the cold-tested magnets being installed in RHIC. The 5 cold-tested D0 magnets not included in $N_{\text {sample }}$ were:

- DRZ114 was only tested for 3 quenches with the last at $6735 \mathrm{~A}$,

- DRZ117 was only tested for 3 quenches with the last at $6585 \mathrm{~A}$,

- DRZ111 had a problem with the test leads,

- DRZ110 had a problem with the test leads,

- DRZ107 had poor performance, but was not installed.

Note that the data shown in Fig. 13 shows the complete history of the trained magnets. It should be noted that DRZ108 trained up to $7112 \mathrm{~A}$, but then had a series of quenches at lower currents after a thermal cycle. For the probability estimate, we have only counted the quenches before the thermal cycle. It may turn out that this particular magnet might need to be replaced for currents above 6000 A. The uninstalled magnet DRZ126 had a good training record and may make a good replacement.
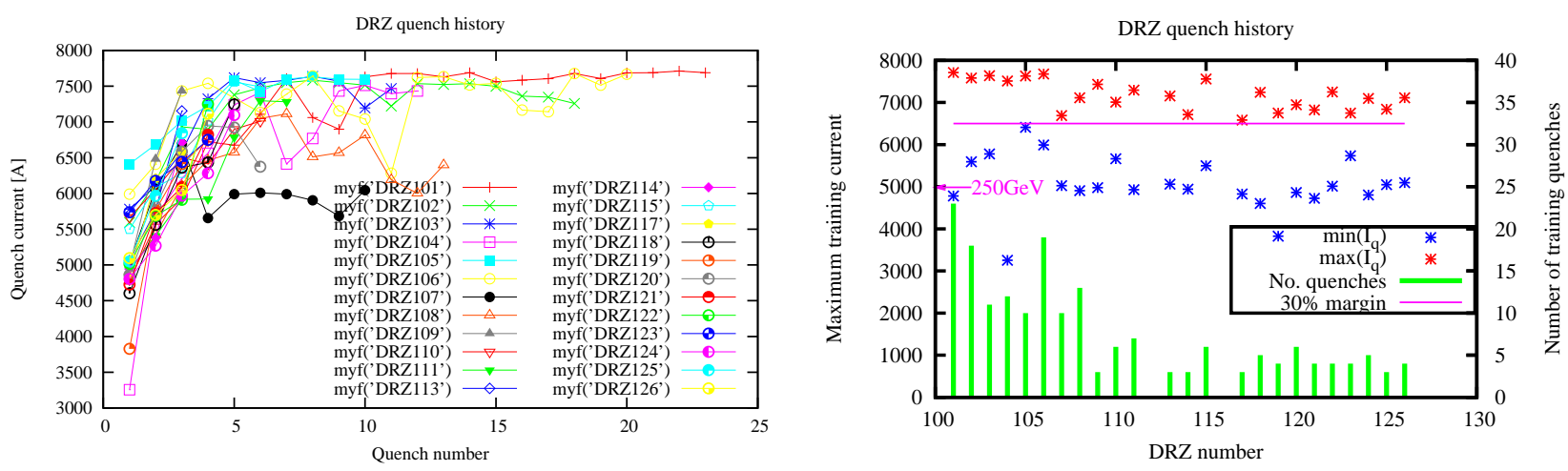

Figure 13. Left: Quench history of D0 magnets tested in the magnet facility. Right: Minimum and Maximum quench currents plotted for the 24 magnets shown in the left plot. A magenta arrow indicates the operating current for $250 \mathrm{GeV}$ with a $0^{\circ}$ crossing angle. The magenta line is drawn for a $30 \%$ increase of current above the nominal $250 \mathrm{GeV}$ value at $5 \mathrm{kA}$. The green histogram indicates the number of times the magnet was quenched in the test facility. Note that DRZ107 and DRZ126 were not installed in RHIC. 

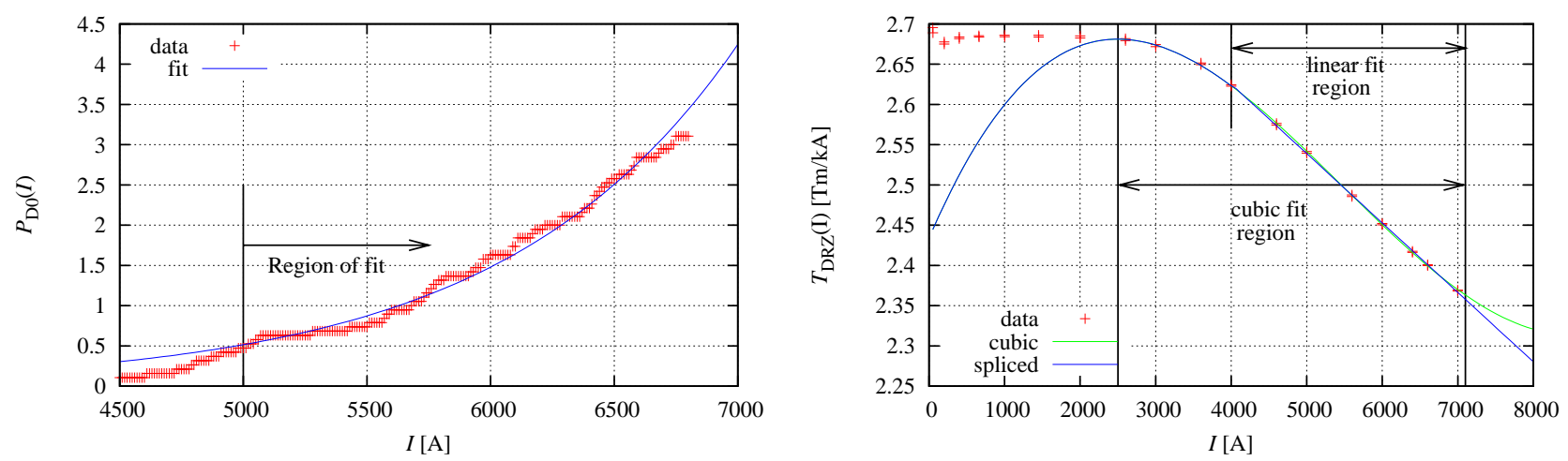

Figure 14. Left: Probability $P_{\mathrm{D} 0}(I)$ of a quench of a D0 magnet trained up to a given current. Since we have operated the D0 magnets at $5000 \mathrm{~A}$ in RHIC with no training quenches, this probability curve is clearly an over estimate and should have an offset subtracted. Right: Fit of transfer function to data ${ }^{\dagger}$ for magnets DRZ105 and DRZ106. A cubic polynomial was spliced to a linear function to approximate the transfer function above $2500 \mathrm{~A}$.

Figs. 14 shows the estimation of the quench probability function for the $N_{\text {sample }}$ D0 magnets. It should be noted that the probability for an untested magnet to have one training quench to reach $5000 \mathrm{~A}$ is $0.47 \pm 0.16$; however all 24 installed magnets have been operated at $4988 \mathrm{~A}$ in RHIC without quenching at some time in the last 10 years. The red curve in Fig. 15 shows the estimated number of training quenches of D0 magnets we could expect if none of the magnets retained their previous training past $5000 \mathrm{~A}$. This was calculated as for the previous magnet types, except that the probability function $P_{D 0}(I)$ was replaced by $P_{D 0}(I)-P_{D 0}\left(I_{250}\right)$. The lower estimate (blue curve) assuming total retention of training was calculated by replacing the probability function for the $j^{\text {th }}$ magnet by $P_{D 0}(I)-P_{D 0}\left(I_{\mathrm{qmax}, j}\right)$ where $I_{\mathrm{qmax}, j}$ was the maximum training current for the $j^{\text {th }}$ magnet. While these curves give a wide variation for the number of expected training quenches, we expect most of the previous training to be retained, so the lower (blue) curve is probably a better estimate.

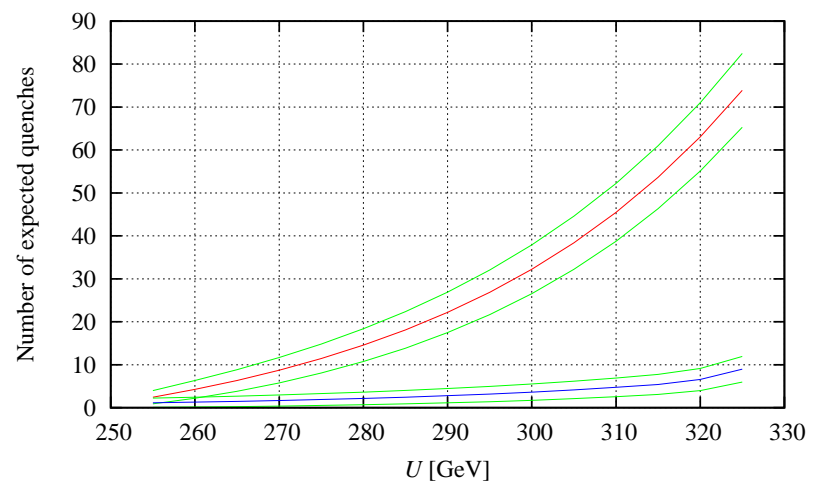

Figure 15. Estimated number of training quenches versus energy for the D0 magnets. The red curve is for the assumption that none of the magnets retained any previous training beyond $5000 \mathrm{~A}$; whereas the blue curve is for total retention of previous training.

\subsection{DX dipoles}

A fit of the transfer function for the DX magnets is shown in Fig. 16. Since the magnets require some retraining after a thermal cycle, we did not calculate a quench probability function for these magnets, but include the full quench history in Tables $2-4$. For a $0^{\circ}$ crossing angle, at $250 \mathrm{GeV}$, the DX magnets need to operate at about $6415 \mathrm{~A}$. While the short-sample current for the DX magnets was estimated to be around $8300 \mathrm{~A}$ at $4.35 \mathrm{~K}$, the actual performance is not that good. The forces are large enough to cause conductor motion well below the

$\dagger$ It is interesting to note that this data shows an inflection point point between 4000 and $7000 \mathrm{~A}$. If a magnet is driven far into saturation, the transfer function will in fact change to a positive curvature since the relative permeability must asymptotically approach one. 
short sample current, and thermal cycling requires a number of retraining quenches for some of the installed 12 magnets to reach the 6415 A values. Since we presently only collide at IR's 6 and 8 , the required currents can be lower at the other four IR's by allowing nonzero crossing angles.

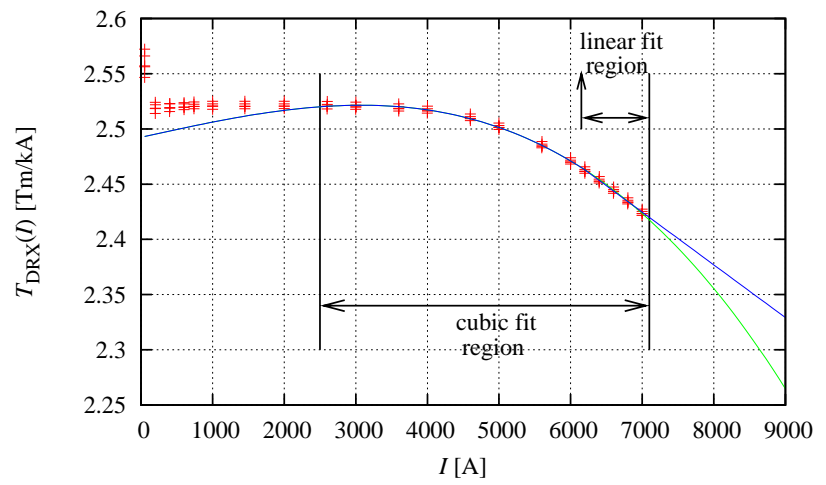

Figure 16. Fit of transfer function to data for magnets DRX106, DRX107, DRX110, DRX112, and DRX113. A cubic polynomial was spliced to a linear function to approximate the transfer function above $2500 \mathrm{~A}$. 
Table 2. Quench history for DX magnets at STAR and PHENIX with $0^{\circ}$-crossing angles.

\begin{tabular}{|c|c|c|c|c|c|c|c|}
\hline Location & Serial \# & Year & Run & sequence & $I_{\mathrm{q}}[\mathrm{A}]^{\mathrm{a}}$ & $I_{\mathrm{op}}[\mathrm{A}]^{\mathrm{b}}$ & $\operatorname{margin}[\%]^{\mathrm{c}}$ \\
\hline g5-dhx & DRX109 & 1999 & -1 & 1 & 6286 & 6409 & -1.92 \\
\hline g5-dhx & DRX109 & 1999 & -1 & 2 & 6700 & 6409 & 4.54 \\
\hline g5-dhx & DRX109 & 1999 & -1 & 3 & 6844 & 6409 & 6.79 \\
\hline g5-dhx & DRX109 & 1999 & -1 & 4 & 7010 & 6409 & 9.38 \\
\hline g5-dhx & DRX109 & 1999 & -1 & 5 & 7137 & 6409 & 11.36 \\
\hline g5-dhx & DRX109 & 1999 & -1 & 6 & 7291 & 6409 & 13.77 \\
\hline g5-dhx & DRX109 & 1999 & -1 & 7 & 7418 & 6409 & 15.75 \\
\hline g5-dhx & DRX109 & 1999 & -1 & 8 & $\geq 7500$ & 6409 & 17.03 \\
\hline g5-dhx & DRX109 & 2000 & 1 & 9 & 6007 & 6409 & -6.27 \\
\hline g5-dhx & DRX109 & 2001 & 2 & 10 & 6289 & 6409 & -1.87 \\
\hline g6-dhx & DRX108 & 1999 & -1 & 1 & 4848 & 6409 & -24.35 \\
\hline g6-dhx & DRX108 & 1999 & -1 & 2 & 6076 & 6409 & -5.19 \\
\hline g6-dhx & DRX108 & 1999 & -1 & 3 & 6363 & 6409 & -0.71 \\
\hline g6-dhx & DRX108 & 1999 & -1 & 4 & 6930 & 6409 & 8.13 \\
\hline g6-dhx & DRX108 & 1999 & -1 & 5 & 7078 & 6409 & 10.44 \\
\hline g6-dhx & DRX108 & 1999 & -1 & 6 & 7349 & 6409 & 14.67 \\
\hline g6-dhx & DRX108 & 1999 & -1 & 7 & $\geq 7500$ & 6409 & 17.03 \\
\hline g6-dhx & DRX108 & 2001 & 2 & 8 & 6396 & 6409 & -0.20 \\
\hline g6-dhx & DRX108 & 2009 & 10 & 9 & 6393 & 6409 & -0.25 \\
\hline g7-dhx & DRX110 & 1999 & -1 & 1 & 5992 & 6406 & -6.46 \\
\hline g7-dhx & DRX110 & 1999 & -1 & 2 & 6355 & 6406 & -0.79 \\
\hline g7-dhx & DRX110 & 1999 & -1 & 3 & 6984 & 6406 & 9.03 \\
\hline g7-dhx & DRX110 & 1999 & -1 & 4 & 6940 & 6406 & 8.34 \\
\hline g7-dhx & DRX110 & 1999 & -1 & 5 & 6950 & 6406 & 8.50 \\
\hline g7-dhx & DRX110 & 1999 & -1 & 6 & 7158 & 6406 & 11.74 \\
\hline g7-dhx & DRX110 & 1999 & -1 & 7 & 7235 & 6406 & 12.95 \\
\hline g7-dhx & DRX110 & 1999 & -1 & 8 & 7254 & 6406 & 13.24 \\
\hline g7-dhx & DRX110 & 1999 & -1 & 9 & 7287 & 6406 & 13.76 \\
\hline g7-dhx & DRX110 & 1999 & -1 & 10 & 7466 & 6406 & 16.55 \\
\hline g7-dhx & DRX110 & 1999 & -1 & 11 & 7483 & 6406 & 16.82 \\
\hline g7-dhx & DRX110 & 1999 & -1 & 12 & $\geq 7500$ & 6406 & 17.08 \\
\hline g7-dhx & DRX110 & 2001 & 2 & 13 & 5679 & 6406 & -11.34 \\
\hline g7-dhx & DRX110 & 2001 & 2 & 14 & 6189 & 6406 & -3.38 \\
\hline g7-dhx & DRX110 & 2002 & 3 & 15 & 5556 & 6406 & -13.26 \\
\hline g7-dhx & DRX110 & 2003 & 4 & 16 & 5529 & 6406 & -13.69 \\
\hline g7-dhx & DRX110 & 2004 & 5 & 17 & 5454 & 6406 & -14.86 \\
\hline g7-dhx & DRX110 & 2006 & 6 & 18 & 5366 & 6406 & -16.23 \\
\hline g7-dhx & DRX110 & 2006 & 6 & 19 & 6082 & 6406 & -5.05 \\
\hline g7-dhx & DRX110 & 2009 & 9 & 20 & 5978 & 6406 & -6.68 \\
\hline g8-dhx & DRX111 & 1999 & -1 & 1 & 6231 & 6406 & -2.73 \\
\hline g8-dhx & DRX111 & 1999 & -1 & 2 & 6370 & 6406 & -0.56 \\
\hline g8-dhx & DRX111 & 1999 & -1 & 3 & 6692 & 6406 & 4.47 \\
\hline g8-dhx & DRX111 & 1999 & -1 & 4 & 7163 & 6406 & 11.82 \\
\hline g8-dhx & DRX111 & 1999 & -1 & 5 & 7222 & 6406 & 12.74 \\
\hline g8-dhx & DRX111 & 1999 & -1 & 6 & 7389 & 6406 & 15.35 \\
\hline g8-dhx & DRX111 & 1999 & -1 & 7 & 7488 & 6406 & 16.90 \\
\hline g8-dhx & DRX111 & 2006 & 6 & 8 & 6272 & 6406 & -2.09 \\
\hline g8-dhx & DRX111 & 2009 & 9 & 9 & 6292 & 6406 & -1.78 \\
\hline g8-dhx & DRX111 & 2009 & 10 & 10 & 5971 & 6406 & -6.79 \\
\hline g8-dhx & DRX111 & 2009 & 10 & 11 & 6365 & 6406 & -0.64 \\
\hline
\end{tabular}

a) $\geq 7500$ indicates that the magnet ramped up to $7500 \mathrm{~A}$ without quenching.

b) Operating current for stored beam at $250 \mathrm{GeV}$ in FY09 fill 10348.

c) $\operatorname{margin}=\left(I_{\mathrm{q}}-I_{\mathrm{op}}\right) / I_{\mathrm{op}}$. 
Table 3. Quench history for DX magnets at IP's 2 and 10 with nozero crossing angles. ${ }^{\dagger}$

\begin{tabular}{|c|c|c|c|c|c|c|c|}
\hline Location & Serial \# & Year & Run & sequence & $I_{\mathrm{q}}[\mathrm{A}]^{\mathrm{a}}$ & $I_{\mathrm{op}}[\mathrm{A}]^{\mathrm{b}}$ & $\operatorname{margin}[\%]^{\mathrm{c}}$ \\
\hline g1-dhx & DRX107 & 1999 & -1 & 1 & 5646 & 5628 & 0.32 \\
\hline g1-dhx & DRX107 & 1999 & -1 & 2 & 6453 & 5628 & 14.66 \\
\hline g1-dhx & DRX107 & 1999 & -1 & 3 & 6624 & 5628 & 17.69 \\
\hline g1-dhx & DRX107 & 1999 & -1 & 4 & 6743 & 5628 & 19.81 \\
\hline g1-dhx & DRX107 & 1999 & -1 & 5 & 6844 & 5628 & 21.60 \\
\hline g1-dhx & DRX107 & 1999 & -1 & 6 & 7097 & 5628 & 26.10 \\
\hline g1-dhx & DRX107 & 1999 & -1 & 7 & 7333 & 5628 & 30.29 \\
\hline g1-dhx & DRX107 & 1999 & -1 & 8 & $\geq 7500$ & 5628 & 33.26 \\
\hline g1-dhx & DRX107 & 2001 & 2 & 9 & 5816 & 5628 & 3.34 \\
\hline g1-dhx & DRX107 & 2001 & 2 & 10 & 6373 & 5628 & 13.23 \\
\hline g1-dhx & DRX107 & 2003 & 4 & 11 & 5839 & 5628 & 3.75 \\
\hline g1-dhx & DRX107 & 2003 & 4 & 12 & 6047 & 5628 & 7.44 \\
\hline g1-dhx & DRX107 & 2004 & 5 & 13 & 4626 & 5628 & -17.81 \\
\hline g1-dhx & DRX107 & 2006 & 6 & 14 & 5958 & 5628 & 5.86 \\
\hline g2-dhx & DRX106 & 1999 & -1 & 1 & 5461 & 5628 & -2.97 \\
\hline g2-dhx & DRX106 & 1999 & -1 & 2 & 6130 & 5628 & 8.92 \\
\hline g2-dhx & DRX106 & 1999 & -1 & 3 & 6491 & 5628 & 15.33 \\
\hline g2-dhx & DRX106 & 1999 & -1 & 4 & 6758 & 5628 & 20.08 \\
\hline g2-dhx & DRX106 & 1999 & -1 & 5 & 6896 & 5628 & 22.53 \\
\hline g2-dhx & DRX106 & 1999 & -1 & 6 & 6995 & 5628 & 24.29 \\
\hline g2-dhx & DRX106 & 1999 & -1 & 7 & 7152 & 5628 & 27.08 \\
\hline g2-dhx & DRX106 & 1999 & -1 & 8 & 7260 & 5628 & 28.99 \\
\hline g2-dhx & DRX106 & 1999 & -1 & 9 & 7499 & 5628 & 33.24 \\
\hline g2-dhx & DRX106 & 1999 & -1 & 10 & $\geq 7500$ & 5628 & 33.26 \\
\hline g2-dhx & DRX106 & 2000 & 1 & 11 & 6136 & 5628 & 9.02 \\
\hline g9-dhx & DRX112 & 1999 & -1 & 1 & 6286 & 5629 & 11.68 \\
\hline g9-dhx & DRX112 & 1999 & -1 & 2 & 6844 & 5629 & 21.59 \\
\hline g9-dhx & DRX112 & 1999 & -1 & 3 & 7082 & 5629 & 25.82 \\
\hline g9-dhx & DRX112 & 1999 & -1 & 4 & 7301 & 5629 & 29.71 \\
\hline g9-dhx & DRX112 & 1999 & -1 & 5 & 7330 & 5629 & 30.23 \\
\hline g9-dhx & DRX112 & 1999 & -1 & 6 & 7500 & 5629 & 33.25 \\
\hline g9-dhx & DRX112 & 1999 & -1 & 7 & $\geq 7500$ & 5629 & 33.25 \\
\hline g9-dhx & DRX112 & 2001 & 2 & 8 & 6256 & 5629 & 11.15 \\
\hline g9-dhx & DRX112 & 2003 & 4 & 9 & 5954 & 5629 & 5.78 \\
\hline g10-dhx & DRX113 & 1999 & -1 & 1 & 5689 & 5618 & 1.26 \\
\hline g10-dhx & DRX113 & 1999 & -1 & 2 & 6449 & 5618 & 14.79 \\
\hline g10-dhx & DRX113 & 1999 & -1 & 3 & 7041 & 5618 & 25.33 \\
\hline g10-dhx & DRX113 & 1999 & -1 & 4 & 7249 & 5618 & 29.03 \\
\hline g10-dhx & DRX113 & 1999 & -1 & 5 & 7497 & 5618 & 33.44 \\
\hline g10-dhx & DRX113 & 1999 & -1 & 6 & $\geq 7500$ & 5618 & 33.50 \\
\hline g10-dhx & DRX113 & 2000 & 1 & 7 & 5895 & 5618 & 4.93 \\
\hline g10-dhx & DRX113 & 2000 & 1 & 8 & 6336 & 5618 & 12.78 \\
\hline
\end{tabular}

$\dagger$ The crossing angle was approximately $2.3 \mathrm{mr}$ for these two interaction points.

a) $\geq 7500$ indicates that the magnet ramped up to $7500 \mathrm{~A}$ without quenching.

b) Operating current for stored beam at $250 \mathrm{GeV}$ in FY09 fill 10348.

c) $\operatorname{margin}=\left(I_{\mathrm{q}}-I_{\mathrm{op}}\right) / I_{\mathrm{op}}$. 
Table 4. Quench history for DX magnets at IP's 4 and 12 with $0^{\circ}$-crossing angles.

\begin{tabular}{|c|c|c|c|c|c|c|c|}
\hline Location & Serial \# & Year & Run & sequence & $I_{\mathrm{q}}[\mathrm{A}]^{\mathrm{a}}$ & $I_{\mathrm{op}}[\mathrm{A}]^{\mathrm{b}}$ & $\operatorname{margin}[\%]^{\mathrm{c}}$ \\
\hline g3-dhx & DRX102 & 1999 & -1 & 1 & 6004 & 6403 & -6.23 \\
\hline g3-dhx & DRX102 & 1999 & -1 & 2 & 7124 & 6403 & 11.26 \\
\hline g3-dhx & DRX102 & 1999 & -1 & 3 & 7469 & 6403 & 16.65 \\
\hline g3-dhx & DRX102 & 1999 & -1 & 4 & $\geq 7500$ & 6403 & 17.13 \\
\hline g3-dhx & DRX102 & 1999 & -1 & 5 & $\geq 7500$ & 6403 & 17.13 \\
\hline g3-dhx & DRX102 & 1999 & -1 & 6 & $\geq 7500$ & 6403 & 17.13 \\
\hline g3-dhx & DRX102 & 1999 & -1 & 7 & $\geq 7500$ & 6403 & 17.13 \\
\hline g3-dhx & DRX102 & 1999 & -1 & 8 & 7375 & 6403 & 15.18 \\
\hline g4-dhx & DRX103 & 1999 & -1 & 1 & 6465 & 6403 & 0.97 \\
\hline g4-dhx & DRX103 & 1999 & -1 & 2 & 6789 & 6403 & 6.03 \\
\hline g4-dhx & DRX103 & 1999 & -1 & 3 & 6908 & 6403 & 7.88 \\
\hline g4-dhx & DRX103 & 1999 & -1 & 4 & 7054 & 6403 & 10.16 \\
\hline g4-dhx & DRX103 & 1999 & -1 & 5 & 7263 & 6403 & 13.43 \\
\hline g4-dhx & DRX103 & 2000 & 1 & 6 & 5839 & 6403 & -8.81 \\
\hline g4-dhx & DRX103 & 2001 & 2 & 7 & 5766 & 6403 & -9.95 \\
\hline g4-dhx & DRX103 & 2003 & 4 & 8 & 6223 & 6403 & -2.81 \\
\hline g4-dhx & DRX103 & 2003 & 4 & 9 & 6249 & 6403 & -2.41 \\
\hline g4-dhx & DRX103 & 2006 & 6 & 10 & 6120 & 6403 & -4.42 \\
\hline g4-dhx & DRX103 & 2007 & 8 & 11 & 6443 & 6403 & 0.62 \\
\hline g4-dhx & DRX103 & 2009 & 9 & 12 & 6078 & 6403 & -5.08 \\
\hline g4-dhx & DRX103 & 2009 & 9 & 13 & 6412 & 6403 & 0.14 \\
\hline g11-dhx & DRX104 & 1999 & -1 & 1 & 6133 & 6415 & -4.39 \\
\hline g11-dhx & DRX104 & 1999 & -1 & 2 & 6870 & 6415 & 7.10 \\
\hline g11-dhx & DRX104 & 1999 & -1 & 3 & 7015 & 6415 & 9.36 \\
\hline g11-dhx & DRX104 & 1999 & -1 & 4 & 7267 & 6415 & 13.29 \\
\hline g11-dhx & DRX104 & 1999 & -1 & 5 & 7353 & 6415 & 14.63 \\
\hline g11-dhx & DRX104 & 1999 & -1 & 6 & 7495 & 6415 & 16.84 \\
\hline g11-dhx & DRX104 & 1999 & -1 & 7 & $\geq 7500$ & 6415 & 16.92 \\
\hline g11-dhx & DRX104 & 2001 & 2 & 8 & 6324 & 6415 & -1.41 \\
\hline g11-dhx & DRX104 & 2001 & 2 & 9 & 6367 & 6415 & -0.74 \\
\hline g11-dhx & DRX104 & 2003 & 4 & 10 & 5981 & 6415 & -6.76 \\
\hline g11-dhx & DRX104 & 2003 & 4 & 11 & 6282 & 6415 & -2.07 \\
\hline g11-dhx & DRX104 & 2003 & 4 & 12 & 6120 & 6415 & -4.59 \\
\hline g11-dhx & DRX104 & 2003 & 4 & 13 & 6150 & 6415 & -4.13 \\
\hline g11-dhx & DRX104 & 2003 & 4 & 14 & 6238 & 6415 & -2.75 \\
\hline g11-dhx & DRX104 & 2006 & 6 & 15 & 6355 & 6415 & -0.93 \\
\hline g11-dhx & DRX104 & 2009 & 9 & 16 & 6264 & 6415 & -2.35 \\
\hline g11-dhx & DRX104 & 2009 & 9 & 17 & 6408 & 6415 & -0.10 \\
\hline g12-dhx & DRX105 & 1999 & -1 & 1 & 6083 & 6415 & -5.17 \\
\hline g12-dhx & DRX105 & 1999 & -1 & 2 & 6720 & 6415 & 4.76 \\
\hline g12-dhx & DRX105 & 1999 & -1 & 3 & 6877 & 6415 & 7.21 \\
\hline g12-dhx & DRX105 & 1999 & -1 & 4 & 7068 & 6415 & 10.19 \\
\hline g12-dhx & DRX105 & 1999 & -1 & 5 & 7333 & 6415 & 14.32 \\
\hline g12-dhx & DRX105 & 2006 & 6 & 6 & 6365 & 6415 & -0.77 \\
\hline
\end{tabular}

a) $\geq 7500$ indicates that the magnet ramped up to $7500 \mathrm{~A}$ without quenching.

b) Operating current for stored beam at $250 \mathrm{GeV}$ in FY09 fill 10348.

c) $\operatorname{margin}=\left(I_{\mathrm{q}}-I_{\mathrm{op}}\right) / I_{\mathrm{op}}$. 


\subsection{CQSSextipole magnets and other correctors}

Table 5. Quench limits for steering, sextipole and other corrector magnets.

\begin{tabular}{|c|c|c|c|c|c|}
\hline Type & $\begin{array}{c}I_{\max }[\mathrm{A}] \\
(\text { Fill 10348) }\end{array}$ & $\begin{array}{c}I_{\mathrm{qmin}}[\mathrm{A}] \\
\text { 1st quench }\end{array}$ & $\begin{array}{l}I_{\text {plat }}[A] \\
\text { plateau }\end{array}$ & $\begin{array}{c}\text { PS rating } \\
{[\mathrm{A}]}\end{array}$ & $I_{\mathrm{Q}}[\mathrm{A}]^{*}$ \\
\hline th & 32.29 & & & 50 & 160 \\
\hline tv & 44.85 & & & 50 & 160 \\
\hline tq & 99.68 & & & 150 & 200 \\
\hline qgt & 41.78 & & & 100 & 190 \\
\hline qs & 52.54 & & & $50 / 60$ & 190 \\
\hline sxf & 12.70 & 91.9 & $>160$ & $33^{\dagger} / 100$ & 200 \\
\hline sxd & 23.70 & 127.0 & $>160$ & $33^{\dagger} / 100$ & 200 \\
\hline $\mathrm{sx} 3$ & 6.67 & & & 50 & \\
\hline oct & 0.16 & & & 50 & 198 \\
\hline dec & 0.14 & & & 50 & 202 \\
\hline dod & 0.05 & & & 50 & \\
\hline
\end{tabular}

* These estimated quench limits $I_{\mathrm{Q}}$ are from the "RHIC Design Manual" [6].

$\dagger$ Note that the RHICgddb..ps_data.I_rating column has the DCCT limit of $75 \mathrm{~A}$ rather than the power supply limit for the 33 A supplies.

Table 5 lists maximum currents for the tq (QRT) and other types of correctors. From maximum operating currents of Fill 10348, the only magnets which appear to exceed the $30 \%$ margin are some steering correctors and skew quadrupoles. (The transition quadrupoles were only used near injection in Fill 10348.) The large currents in some steering correctors may be due either to reversed signs for BPM offsets which were corrected in a later run, or to misaligned magnets. In principle, quadrupole magnets near any limiting steering elements could be realigned to reduce the required currents.

The limits for some skew quadrupoles may need further investigation as to whether their power supplies and feedthroughs should need any upgrading. 
Table 6. Power supply and feedthrough margins for the dipole magnets ordered by PS margin.

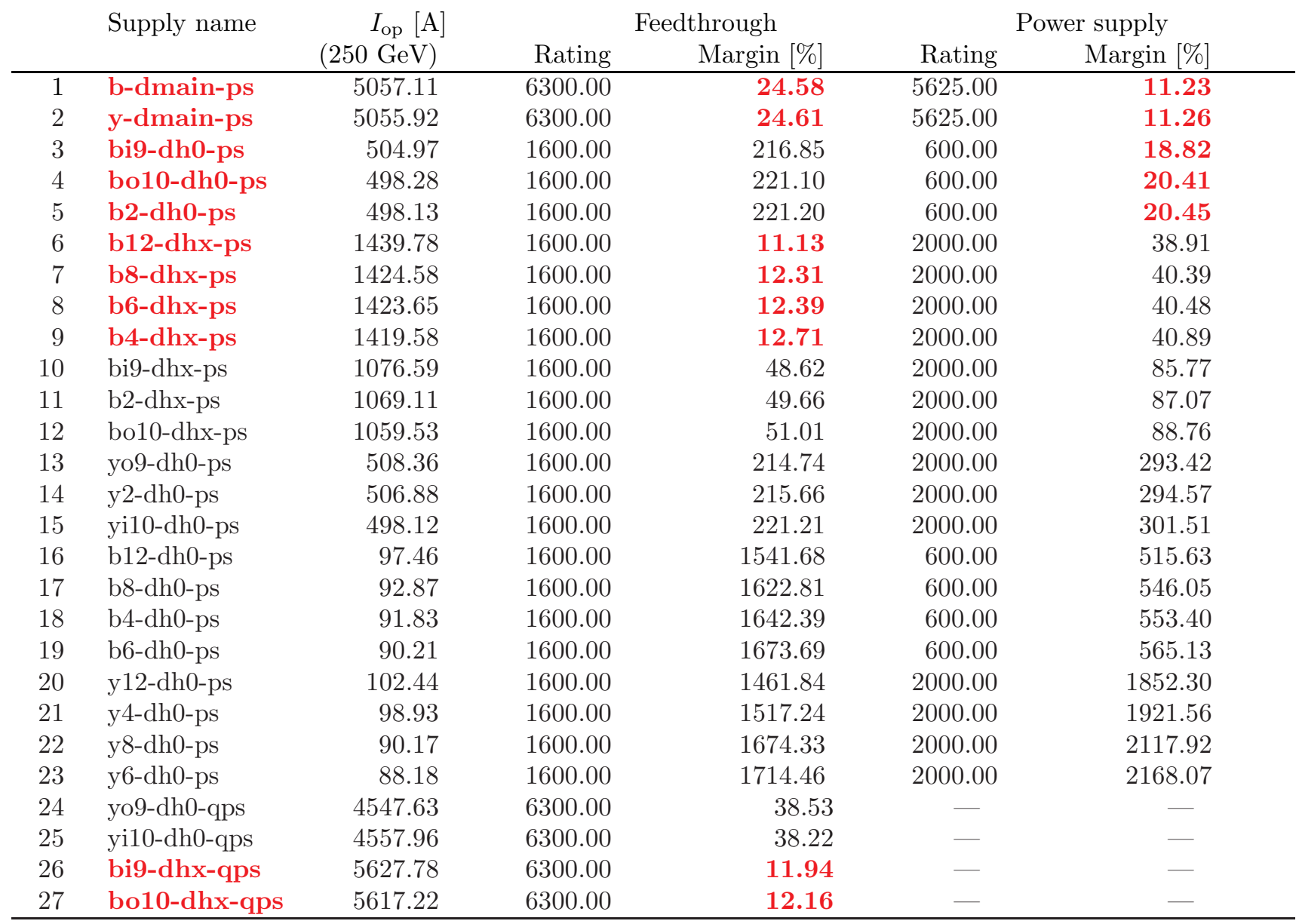

\section{Feedthroughs and power supplies}

In addition to the limits of the magnets, we must consider the limits of power supplies and cryogenic feedthroughs. Table 6 lists the current ratings of the main dipole buss and shunt supplies and feedthroughs for the bending magnets. The margin in percentages above the $250 \mathrm{GeV}$ operating currents are given for both supplies and feedthroughs with margins lower than $30 \%$ highlighted in red. Note that the last two $6300 \mathrm{~A}$ feedthroughs in this table must carry the net current from the quench protection switches and D0 and DX (blue ring only) supplies. According to George Ganetis, these leads should be able to exceed 6300 A by at least $10 \%$ with more gas flow provided that they do not ice up too much on top. (In 2004 PHOBOS ran with around $6400 \mathrm{~A}$ in these leads with a $0^{\circ}$ crossing angle for gold-gold collisions at $100 \mathrm{GeV} / \mathrm{n}$ per beam.)

The next two tables show similar data for the quadrupole supplies and feedthroughs with margins less than $35 \%$; Table 7 is ordered by increasing feedthrough margin, and Table 8 is ordered by increasing power supply margin.

Tables 9-14 show the power supply and feedthrough margins for all main quadrupoles around each of the IR's. 
Table 7. Power supply and feedthrough margins for quads with margins $<35 \%$ order by FT margin.

\begin{tabular}{|c|c|c|c|c|c|c|}
\hline \multirow{2}{*}{\multicolumn{2}{|c|}{ Supply name }} & \multirow{2}{*}{$\begin{array}{c}I_{\mathrm{op}}[\mathrm{A}] \\
(250 \mathrm{GeV})\end{array}$} & \multicolumn{2}{|c|}{ Feedthrough } & \multicolumn{2}{|c|}{ Power supply } \\
\hline & & & Rating & Margin [\%] & Rating & Margin [\%] \\
\hline 1 & y6-q6-ps & 429.96 & 450.00 & 4.66 & 450.00 & 4.66 \\
\hline 2 & b6-q6-ps & 429.84 & 450.00 & 4.69 & 450.00 & 4.69 \\
\hline 3 & y8-q6-ps & 429.75 & 450.00 & 4.71 & 450.00 & 4.71 \\
\hline 4 & b8-q6-ps & 429.67 & 450.00 & 4.73 & 450.00 & 4.73 \\
\hline 5 & yo4-qf8-ps & 140.61 & 150.00 & 6.67 & 200.00 & 42.23 \\
\hline 6 & уо9-qd3-ps & 280.86 & 300.00 & 6.81 & 300.00 & 6.81 \\
\hline 7 & yi10-qf3-ps & 280.46 & 300.00 & 6.97 & 300.00 & 6.97 \\
\hline 8 & уо9-qf8-ps & 140.18 & 150.00 & 7.00 & 200.00 & 42.67 \\
\hline 9 & уо4-qd3-ps & 280.16 & 300.00 & 7.08 & 300.00 & 7.08 \\
\hline 10 & bo10-qd3-ps & 280.08 & 300.00 & 7.11 & 300.00 & 7.11 \\
\hline 11 & bi9-qf3-ps & 280.02 & 300.00 & 7.14 & 300.00 & 7.14 \\
\hline 12 & bo11-qd3-ps & 279.98 & 300.00 & 7.15 & 300.00 & 7.15 \\
\hline 13 & bi12-qf3-ps & 279.64 & 300.00 & 7.28 & 300.00 & 7.28 \\
\hline 14 & yo12-qd3-ps & 279.63 & 300.00 & 7.29 & 300.00 & 7.29 \\
\hline 15 & bi4-qf3-ps & 279.62 & 300.00 & 7.29 & 300.00 & 7.29 \\
\hline 16 & yi11-qf3-ps & 279.61 & 300.00 & 7.29 & 300.00 & 7.29 \\
\hline 17 & yi3-qf3-ps & 279.52 & 300.00 & 7.33 & 300.00 & 7.33 \\
\hline 18 & bo3-qd3-ps & 279.25 & 300.00 & 7.43 & 300.00 & 7.43 \\
\hline 19 & bo3-qf8-ps & 138.33 & 150.00 & 8.44 & 200.00 & 44.59 \\
\hline 20 & yo12-qf8-ps & 137.49 & 150.00 & 9.10 & 200.00 & 45.47 \\
\hline 21 & bo10-qf8-ps & 137.13 & 150.00 & 9.38 & 200.00 & 45.84 \\
\hline 22 & yo1-qf8-ps & 136.50 & 150.00 & 9.89 & 200.00 & 46.52 \\
\hline 23 & bo2-qf8-ps & 135.13 & 150.00 & 11.01 & 200.00 & 48.01 \\
\hline 24 & bo11-qf8-ps & 134.79 & 150.00 & 11.28 & 200.00 & 48.38 \\
\hline 25 & yi3-qf9-ps & 125.57 & 150.00 & 19.45 & 200.00 & 59.27 \\
\hline 26 & bi9-qf9-ps & 123.66 & 150.00 & 21.30 & 200.00 & 61.73 \\
\hline 27 & yi11-qf9-ps & 123.35 & 150.00 & 21.61 & 200.00 & 62.15 \\
\hline 28 & yi10-qf9-ps & 122.70 & 150.00 & 22.25 & 200.00 & 62.99 \\
\hline 29 & yi2-qf9-ps & 122.52 & 150.00 & 22.43 & 200.00 & 63.24 \\
\hline 30 & bi1-qf9-ps & 120.21 & 150.00 & 24.79 & 200.00 & 66.38 \\
\hline 31 & bi4-qf9-ps & 119.04 & 150.00 & 26.01 & 200.00 & 68.01 \\
\hline 32 & bi12-qf9-ps & 118.21 & 150.00 & 26.89 & 200.00 & 69.19 \\
\hline 33 & bi5-qf3-ps & 232.87 & 300.00 & 28.83 & 300.00 & 28.83 \\
\hline 34 & yi7-qf3-ps & 232.23 & 300.00 & 29.18 & 300.00 & 29.18 \\
\hline 35 & yo8-qd3-ps & 232.23 & 300.00 & 29.18 & 300.00 & 29.18 \\
\hline 36 & bi8-qf3-ps & 231.69 & 300.00 & 29.48 & 300.00 & 29.48 \\
\hline 37 & yi6-qf3-ps & 230.87 & 300.00 & 29.94 & 300.00 & 29.94 \\
\hline 38 & bo6-qd3-ps & 229.47 & 300.00 & 30.74 & 300.00 & 30.74 \\
\hline 39 & yo5-qd3-ps & 228.61 & 300.00 & 31.23 & 300.00 & 31.23 \\
\hline 40 & bo7-qd3-ps & 227.78 & 300.00 & 31.71 & 300.00 & 31.71 \\
\hline 41 & b-qmain-ps & 4696.43 & 6300.00 & 34.14 & 5625.00 & 19.77 \\
\hline 42 & y-qmain-ps & 4692.30 & 6300.00 & 34.26 & 5625.00 & 19.88 \\
\hline 43 & b6-q89-ps & 274.04 & 450.00 & 64.21 & 300.00 & 9.47 \\
\hline 44 & b8-q89-ps & 273.37 & 450.00 & 64.61 & 300.00 & 9.74 \\
\hline 45 & y6-q89-ps & 263.74 & 450.00 & 70.62 & 300.00 & 13.75 \\
\hline 46 & y8-q89-ps & 262.68 & 450.00 & 71.31 & 300.00 & 14.21 \\
\hline 47 & yi6-qf1-ps & 165.56 & 300.00 & 81.21 & 200.00 & 20.81 \\
\hline 48 & yi7-qf1-ps & 158.70 & 300.00 & 89.03 & 200.00 & 26.02 \\
\hline 49 & yi2-qf3-ps & 279.50 & 1600.00 & 472.45 & 300.00 & 7.33 \\
\hline 50 & bo2-qd3-ps & 279.47 & 1600.00 & 472.51 & 300.00 & 7.35 \\
\hline 51 & bi1-qf3-ps & 279.42 & 1600.00 & 472.62 & 300.00 & 7.37 \\
\hline 52 & yo1-qd3-ps & 278.58 & 1600.00 & 474.34 & 300.00 & 7.69 \\
\hline
\end{tabular}


Table 8. Power supply and feedthrough margins for quads with margins $<35 \%$ ordered by PS margin.

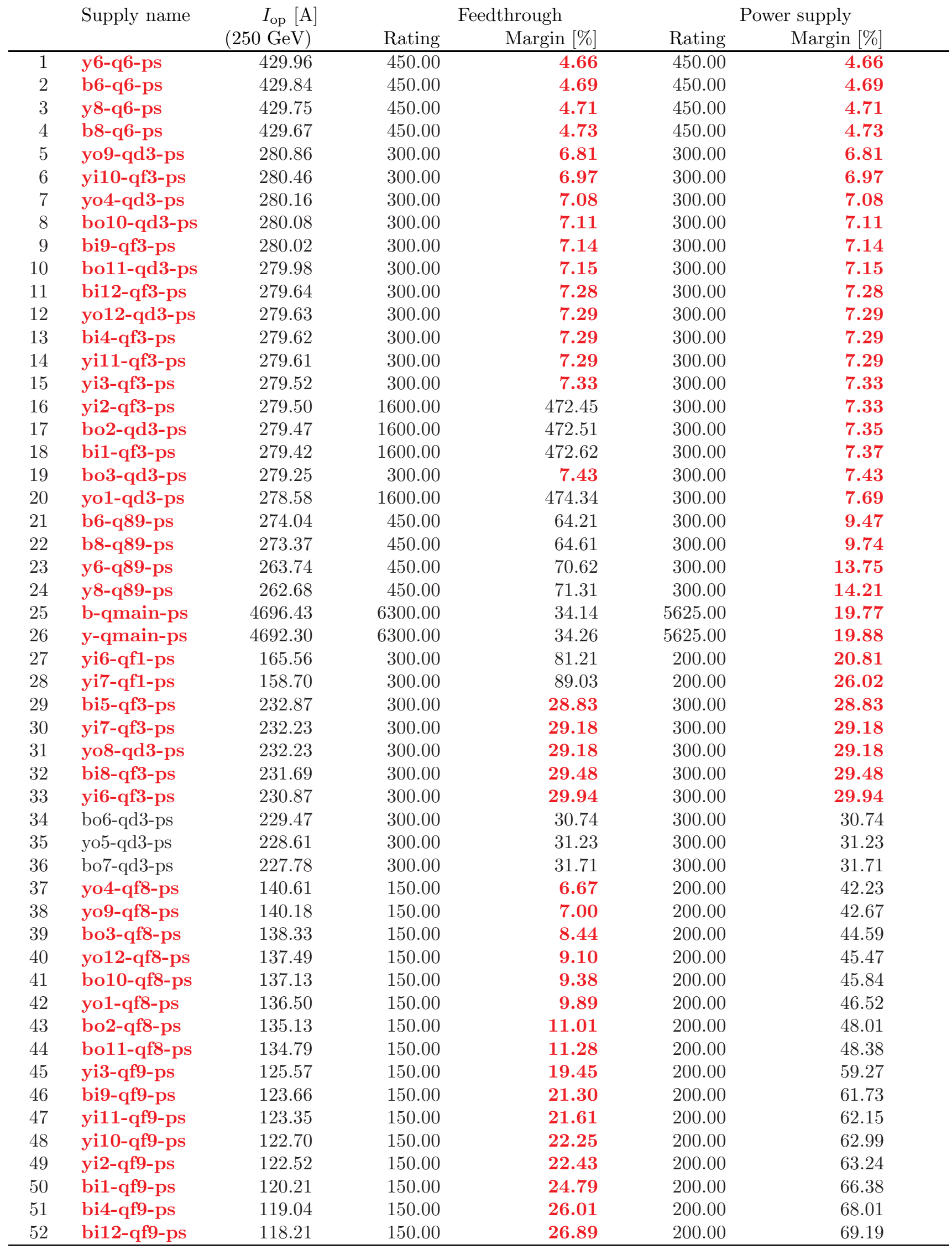


Table 9. Power supply and feedthrough margins for quadrupoles at IR2.

\begin{tabular}{|c|c|c|c|c|c|c|}
\hline & \multirow[t]{2}{*}{ Supply name } & \multirow{2}{*}{$\begin{array}{c}I_{\mathrm{op}}[\mathrm{A}] \\
(250 \mathrm{GeV})\end{array}$} & \multicolumn{2}{|c|}{ Feedthrough } & \multicolumn{2}{|c|}{ Power supply } \\
\hline & & & Rating & Margin [\%] & Rating & Margin [\%] \\
\hline 1 & b2-q6-ps & 33.06 & 450.00 & 1261.00 & 450.00 & 1261.00 \\
\hline 2 & bo2-qf8-ps & 135.13 & 150.00 & 11.01 & 200.00 & 48.01 \\
\hline 3 & bo2-qf2-ps & 93.22 & 150.00 & 60.91 & 150.00 & 60.91 \\
\hline 4 & bo2-qd3-ps & 279.47 & 1600.00 & 472.51 & 300.00 & 7.35 \\
\hline 5 & bo2-qd1-ps & 94.01 & 300.00 & 219.13 & 200.00 & 112.75 \\
\hline 6 & bi1-qf9-ps & 120.21 & 150.00 & 24.79 & 200.00 & 66.38 \\
\hline 7 & bi1-qf3-ps & 279.42 & 1600.00 & 472.62 & 300.00 & 7.37 \\
\hline 8 & bi1-qf1-ps & 113.86 & 300.00 & 163.48 & 200.00 & 75.66 \\
\hline 9 & bi1-qd2-ps & 71.14 & 150.00 & 110.86 & 150.00 & 110.86 \\
\hline 10 & b2-q89-ps & -20.28 & 450.00 & 2119.09 & 300.00 & 1379.39 \\
\hline 11 & b2-q7-ps & 366.55 & 750.00 & 104.61 & 600.00 & 63.69 \\
\hline 12 & yi2-qf9-ps & 122.52 & 150.00 & 22.43 & 200.00 & 63.24 \\
\hline 13 & yo1-qd1-ps & 95.69 & 300.00 & 213.52 & 200.00 & 109.01 \\
\hline 14 & yo1-qd3-ps & 278.58 & 1600.00 & 474.34 & 300.00 & 7.69 \\
\hline 15 & yo1-qf2-ps & 99.67 & 150.00 & 50.50 & 150.00 & 50.50 \\
\hline 16 & yi2-qf3-ps & 279.50 & 1600.00 & 472.45 & 300.00 & 7.33 \\
\hline 17 & yi2-qf1-ps & 93.71 & 300.00 & 220.13 & 200.00 & 113.42 \\
\hline 18 & yi2-qd2-ps & 75.25 & 150.00 & 99.33 & 150.00 & 99.33 \\
\hline 19 & y2-q89-ps & -20.91 & 450.00 & 2052.04 & 300.00 & 1334.69 \\
\hline 20 & y2-q7-ps & 368.16 & 750.00 & 103.72 & 600.00 & 62.97 \\
\hline 21 & y2-q6-ps & 36.01 & 450.00 & 1149.58 & 450.00 & 1149.58 \\
\hline 22 & yo1-qf8-ps & 136.50 & 150.00 & 9.89 & 200.00 & 46.52 \\
\hline
\end{tabular}


Table 10. Power supply and feedthrough margins for quadrupoles at IR4.

\begin{tabular}{|c|c|c|c|c|c|c|}
\hline & \multirow[t]{2}{*}{ Supply name } & \multirow{2}{*}{$\begin{array}{c}I_{\mathrm{op}}[\mathrm{A}] \\
(250 \mathrm{GeV})\end{array}$} & \multicolumn{2}{|c|}{ Feedthrough } & \multicolumn{2}{|c|}{ Power supply } \\
\hline & & & Rating & Margin [\%] & Rating & Margin [\%] \\
\hline 1 & b-qmain-ps & 4696.43 & 6300.00 & 34.14 & 5625.00 & 19.77 \\
\hline 2 & bo3-qf8-ps & 138.33 & 150.00 & 8.44 & 200.00 & 44.59 \\
\hline 3 & bo3-qf6-ps & 38.38 & 450.00 & 1072.59 & 450.00 & 1072.59 \\
\hline 4 & bo3-qf2-ps & 97.54 & 150.00 & 53.78 & 150.00 & 53.78 \\
\hline 5 & bo3-qd7-ps & 361.40 & 750.00 & 107.52 & 600.00 & 66.02 \\
\hline 6 & bo3-qd3-ps & 279.25 & 300.00 & 7.43 & 300.00 & 7.43 \\
\hline 7 & bo3-qd1-ps & 87.35 & 300.00 & 243.45 & 200.00 & 128.97 \\
\hline 8 & bi4-qf9-ps & 119.04 & 150.00 & 26.01 & 200.00 & 68.01 \\
\hline 9 & bi4-qf7-ps & 362.54 & 750.00 & 106.87 & 600.00 & 65.50 \\
\hline 10 & bi4-qf3-ps & 279.62 & 300.00 & 7.29 & 300.00 & 7.29 \\
\hline 11 & bi4-qf1-ps & 70.87 & 300.00 & 323.30 & 200.00 & 182.20 \\
\hline 12 & bi4-qd6-ps & 35.28 & 450.00 & 1175.44 & 450.00 & 1175.44 \\
\hline 13 & bi4-qd2-ps & 95.97 & 150.00 & 56.29 & 150.00 & 56.29 \\
\hline 14 & b4-q89-ps & -20.34 & 450.00 & 2112.59 & 300.00 & 1375.06 \\
\hline 15 & yo4-qd1-ps & 64.16 & 300.00 & 367.60 & 200.00 & 211.74 \\
\hline 16 & yo4-qd3-ps & 280.16 & 300.00 & 7.08 & 300.00 & 7.08 \\
\hline 17 & yo4-qd7-ps & 367.77 & 750.00 & 103.93 & 600.00 & 63.14 \\
\hline 18 & yo4-qf2-ps & 97.07 & 150.00 & 54.52 & 150.00 & 54.52 \\
\hline 19 & yo4-qf6-ps & 35.37 & 450.00 & 1172.22 & 450.00 & 1172.22 \\
\hline 20 & yi3-qf9-ps & 125.57 & 150.00 & 19.45 & 200.00 & 59.27 \\
\hline 21 & yi3-qf7-ps & 366.84 & 750.00 & 104.45 & 600.00 & 63.56 \\
\hline 22 & yi3-qf3-ps & 279.52 & 300.00 & 7.33 & 300.00 & 7.33 \\
\hline 23 & yi3-qf1-ps & 92.73 & 300.00 & 223.52 & 200.00 & 115.68 \\
\hline 24 & yi3-qd6-ps & 35.03 & 450.00 & 1184.78 & 450.00 & 1184.78 \\
\hline 25 & yi3-qd2-ps & 72.52 & 150.00 & 106.84 & 150.00 & 106.84 \\
\hline 26 & y4-q89-ps & -20.63 & 450.00 & 2081.49 & 300.00 & 1354.33 \\
\hline 27 & $y$-qmain-ps & 4692.30 & 6300.00 & 34.26 & 5625.00 & 19.88 \\
\hline 28 & уо4-qf8-ps & 140.61 & 150.00 & 6.67 & 200.00 & 42.23 \\
\hline
\end{tabular}


Table 11. Power supply and feedthrough margins for quadrupoles at IR6.

$\begin{array}{rlrrrrr} & \text { Supply name } & \begin{array}{r}I_{\mathrm{op}}[\mathrm{A}] \\ (250 \mathrm{GeV})\end{array} & \text { Rating } & \begin{array}{r}\text { Feedthrough } \\ \text { Margin [\%] }\end{array} & \text { Rating } & \text { Power supply } \\ \text { Margin [\%] }\end{array}$

Table 12. Power supply and feedthrough margins for quadrupoles at IR8.

\begin{tabular}{rlrrrrr} 
& Supply name & $\begin{array}{r}I_{\mathrm{op}}[\mathrm{A}] \\
(250 \mathrm{GeV})\end{array}$ & Rating & $\begin{array}{r}\text { Feedthrough } \\
\text { Margin [\%] }\end{array}$ & Rating & Power supply \\
& & 429.67 & 450.00 & 4.73 & 450.00 & 4.73 \\
\hline 1 & b8-q6-ps & 71.95 & 150.00 & 108.48 & 200.00 & 177.97 \\
2 & bo7-qf8-ps & 18.48 & 150.00 & 711.56 & 150.00 & 711.56 \\
3 & bo7-qf2-ps & 227.78 & 300.00 & 31.71 & 300.00 & 31.71 \\
4 & bo7-qd3-ps & 128.15 & 300.00 & 134.10 & 200.00 & 56.06 \\
5 & bo7-qd1-ps & 101.12 & 150.00 & 48.34 & 200.00 & 97.78 \\
6 & bi8-qf9-ps & 231.69 & 300.00 & 29.48 & 300.00 & 29.48 \\
7 & bi8-qf3-ps & 132.62 & 300.00 & 126.20 & 200.00 & 50.80 \\
8 & bi8-qf1-ps & 18.61 & 150.00 & 705.84 & 150.00 & 705.84 \\
9 & bi8-qd2-ps & 273.37 & 450.00 & 64.61 & 300.00 & 9.74 \\
10 & b8-q89-ps & 80.00 & 750.00 & 837.54 & 600.00 & 650.03 \\
11 & b8-q7-ps & 97.13 & 150.00 & 54.44 & 200.00 & 105.92 \\
12 & yi7-qf9-ps & 116.48 & 300.00 & 157.56 & 200.00 & 71.71 \\
13 & yo8-qd1-ps & 232.23 & 300.00 & 29.18 & 300.00 & 29.18 \\
14 & yo8-qd3-ps & 26.29 & 150.00 & 470.47 & 150.00 & 470.47 \\
15 & yo8-qf2-ps & 232.23 & 300.00 & 29.18 & 300.00 & 29.18 \\
16 & yi7-qf3-ps & 158.70 & 300.00 & 89.03 & 200.00 & 26.02 \\
17 & yi7-qf1-ps & 21.02 & 150.00 & 613.77 & 150.00 & 613.77 \\
18 & yi7-qd2-ps & 262.68 & 450.00 & 71.31 & 300.00 & 14.21 \\
19 & y8-q89-ps & 117.20 & 750.00 & 539.92 & 600.00 & 411.94 \\
20 & y8-q7-ps & 429.75 & 450.00 & 4.71 & 450.00 & 4.71 \\
21 & y8-q6-ps & 69.87 & 150.00 & 114.70 & 200.00 & 186.26 \\
22 & yo8-qf8-ps & & & & & \\
\hline
\end{tabular}


Table 13. Power supply and feedthrough margins for quadrupoles at IR10.

\begin{tabular}{rlrrrrr} 
& Supply name & $\begin{array}{r}I_{\text {op }}[\mathrm{A}] \\
(250 \mathrm{GeV})\end{array}$ & Rating & $\begin{array}{r}\text { Feedthrough } \\
\text { Margin [\%] }\end{array}$ & Rating & Margin [\%] \\
\hline 1 & b-qtrim-ps & 165.36 & 450.00 & 172.14 & 300.00 & 81.42 \\
2 & bo10-qf8-ps & 137.13 & 150.00 & 9.38 & 200.00 & 45.84 \\
3 & bo10-qf6-ps & 36.44 & 450.00 & 1134.75 & 450.00 & 1134.75 \\
4 & bo10-qf2-ps & 92.78 & 150.00 & 61.68 & 150.00 & 61.68 \\
5 & bo10-qd9-ps & -20.25 & 150.00 & 640.59 & 300.00 & 1381.17 \\
6 & bo10-qd7-ps & 362.91 & 750.00 & 106.66 & 600.00 & 65.33 \\
7 & bo10-qd3-ps & 280.08 & 300.00 & 7.11 & 300.00 & 7.11 \\
8 & bo10-qd1-ps & 98.30 & 300.00 & 205.19 & 200.00 & 103.46 \\
9 & bi9-qf9-ps & 123.66 & 150.00 & 21.30 & 200.00 & 61.73 \\
10 & bi9-q89-ps & -20.15 & 450.00 & 2133.54 & 300.00 & 1389.03 \\
11 & bi9-qd2-ps & 76.14 & 150.00 & 97.00 & 150.00 & 97.00 \\
12 & bi9-qd6-ps & 34.58 & 450.00 & 1201.36 & 450.00 & 1201.36 \\
13 & bi9-qf1-ps & 75.27 & 300.00 & 298.57 & 200.00 & 165.71 \\
14 & bi9-qf3-ps & 280.02 & 300.00 & 7.14 & 300.00 & 7.14 \\
15 & bi9-qf7-ps & 365.12 & 750.00 & 105.41 & 600.00 & 64.33 \\
16 & yo9-qd1-ps & 93.31 & 300.00 & 221.52 & 200.00 & 114.35 \\
17 & yo9-qd3-ps & 280.86 & 300.00 & 6.81 & 300.00 & 6.81 \\
18 & yo9-qd7-ps & 368.54 & 750.00 & 103.51 & 600.00 & 62.81 \\
19 & yo9-qd9-ps & -20.96 & 150.00 & 615.57 & 300.00 & 1331.14 \\
20 & yo9-qf2-ps & 96.62 & 150.00 & 55.25 & 150.00 & 55.25 \\
21 & yo9-qf6-ps & 36.23 & 450.00 & 1141.92 & 450.00 & 1141.92 \\
22 & yi10-qf9-ps & 122.70 & 150.00 & 22.25 & 200.00 & 62.99 \\
23 & yi10-qf7-ps & 367.57 & 750.00 & 104.04 & 600.00 & 63.23 \\
24 & yi10-qf3-ps & 280.46 & 300.00 & 6.97 & 300.00 & 6.97 \\
25 & yi10-qf1-ps & 90.91 & 300.00 & 230.01 & 200.00 & 120.01 \\
26 & yi10-qd6-ps & 36.88 & 450.00 & 1120.04 & 450.00 & 1120.04 \\
27 & yi10-qd2-ps & 75.51 & 150.00 & 98.65 & 150.00 & 98.65 \\
28 & yi10-q89-ps & -20.69 & 450.00 & 2075.06 & 300.00 & 1350.04 \\
29 & y-qtrim-ps & 178.65 & 450.00 & 151.89 & 300.00 & 67.93 \\
30 & yo9-qf8-ps & 140.18 & 150.00 & 7.00 & 200.00 & 42.67 \\
\hline & & & & & \\
\hline
\end{tabular}


Table 14. Power supply and feedthrough margins for quadrupoles at IR12.

\begin{tabular}{rlrrrrr} 
& Supply name & $\begin{array}{r}I_{\text {op }}[\mathrm{A}] \\
(250 \mathrm{GeV})\end{array}$ & Rating & $\begin{array}{r}\text { Feedthrough } \\
\text { Margin [\%] }\end{array}$ & Rating & Mower supply \\
& & 33.96 & 450.00 & 1224.95 & 450.00 & 1224.95 \\
\hline 1 & b12-q6-ps & 134.79 & 150.00 & 11.28 & 200.00 & 48.38 \\
2 & bo11-qf8-ps & 96.92 & 150.00 & 54.77 & 150.00 & 54.77 \\
3 & bo11-qf2-ps & 279.98 & 300.00 & 7.15 & 300.00 & 7.15 \\
4 & bo11-qd3-ps & 92.68 & 300.00 & 223.68 & 200.00 & 115.79 \\
5 & bo11-qd1-ps & 118.21 & 150.00 & 26.89 & 200.00 & 69.19 \\
6 & bi12-qf9-ps & 279.64 & 300.00 & 7.28 & 300.00 & 7.28 \\
7 & bi12-qf3-ps & 95.45 & 300.00 & 214.31 & 200.00 & 109.54 \\
8 & bi12-qf1-ps & 82.81 & 150.00 & 81.14 & 150.00 & 81.14 \\
9 & bi12-qd2-ps & -20.68 & 450.00 & 2076.50 & 300.00 & 1351.00 \\
10 & b12-q89-ps & 363.80 & 750.00 & 106.15 & 600.00 & 64.92 \\
11 & b12-q7-ps & 123.35 & 150.00 & 21.61 & 200.00 & 62.15 \\
12 & yi11-qf9-ps & 84.26 & 300.00 & 256.05 & 200.00 & 137.37 \\
13 & yo12-qd1-ps & 279.63 & 300.00 & 7.29 & 300.00 & 7.29 \\
14 & yo12-qd3-ps & 93.03 & 150.00 & 61.24 & 150.00 & 61.24 \\
15 & yo12-qf2-ps & 279.61 & 300.00 & 7.29 & 300.00 & 7.29 \\
16 & yi11-qf3-ps & 68.90 & 300.00 & 335.40 & 200.00 & 190.27 \\
17 & yi11-qf1-ps & 75.04 & 150.00 & 99.90 & 150.00 & 99.90 \\
18 & yi11-qd2-ps & -20.82 & 450.00 & 2061.51 & 300.00 & 1341.00 \\
19 & y12-q89-ps & 368.58 & 750.00 & 103.48 & 600.00 & 62.79 \\
20 & y12-q7-ps & 34.93 & 450.00 & 1188.23 & 450.00 & 1188.23 \\
21 & y12-q6-ps & 137.49 & 150.00 & 9.10 & 200.00 & 45.47 \\
22 & yo12-qf8-ps & & & & \\
\hline
\end{tabular}

\section{Limited scenario: $45 \%$ increase in $\mathrm{W}$ production rate}
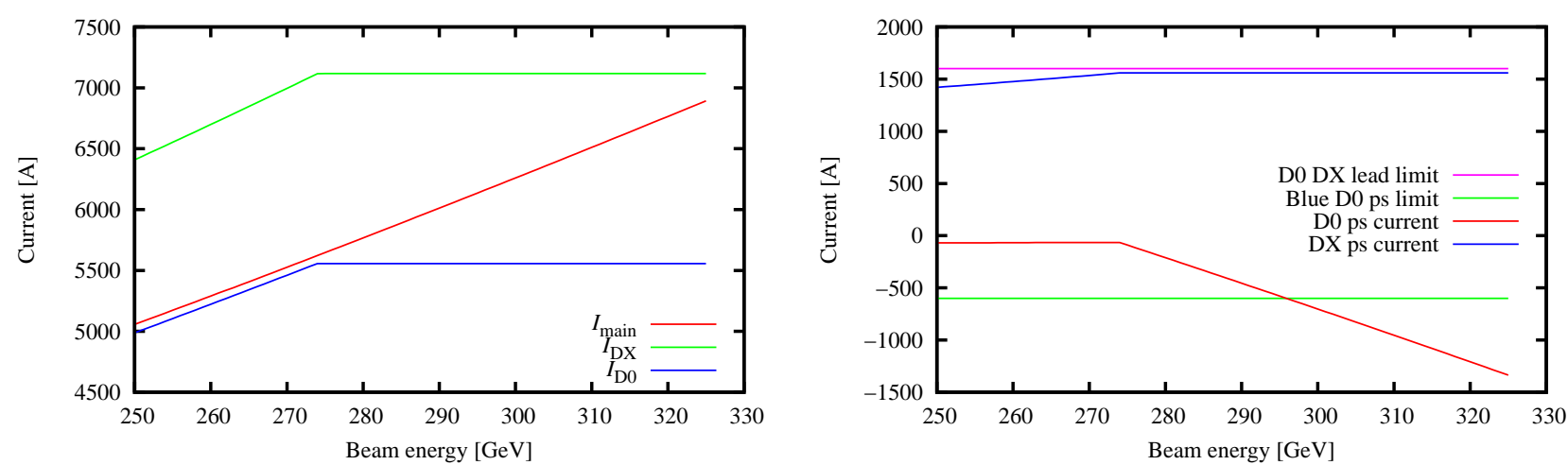

Figure 17. Left: Predicted currents in dipole magnets with a clamp of the DX magnet at 7117 A. Right: Corresponding power supply currents with limits from the cryogenic leads of the DX and D0 shunts at $1600 \mathrm{~A}$ and Blue-ring D0 shunt supplies at $600 \mathrm{~A}$. (As indicated in Table 6, the D0 shunt supplies of the yellow ring supplies limit at 2000 A which is 400 A higher than the D0 shunt leads.)

While the number of new training quenches needed to reach an energy increase to $300 \mathrm{GeV}$ or higher is probably prohibitive, we consider $274 \mathrm{GeV}$ as a more reachable possibility. This value for energy was chosen as the present limit of the main dipole power supplies (5625 A). (According to Carl Schultheiss, it might be possible to raise this power supply limit a little, but the lifetime of the SCR's would be shortened.) In this scenario, we keep the currents for the DX and D0 magnets constant from 250 to $274 \mathrm{GeV}$ at IR's 2, 4, 10, and 12 . At $274 \mathrm{GeV}$ this corresponds to a 2 mrad crossing angle for the four noncolliding IP's (see Fig. 2). Fig. 17 shows the magnet currents (left) and power supply currents (right) for the bending magnets with the present power supply configuration. (Note that the present Blue D0 shunt power supplies would reach their limit at $300 \mathrm{GeV}$.) 

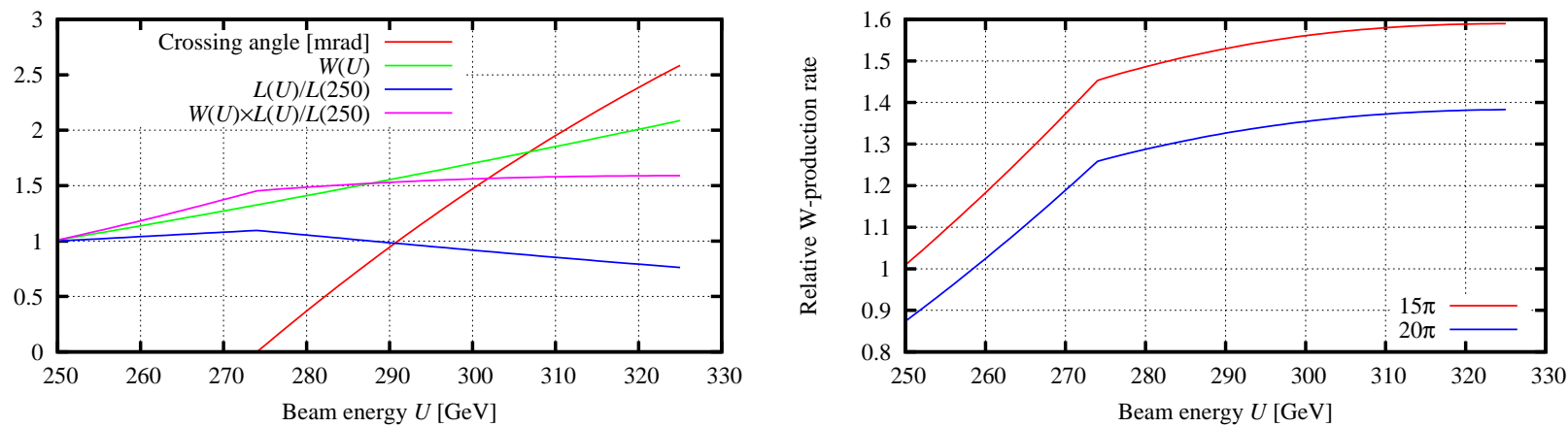

Figure 18. Left: Relative W-production rate vs energy for colliding beams with normalized $95 \%$ emittances of $15 \pi \mu \mathrm{m}$. The relative cross section (in green) from Fig. 1 is combined with the estimated luminosity vs energy (in blue) and adjusted for the crossing angle (in red) to show how the production rate (in magenta) would increase. For this calculation, when the DX magnet current reached $7117 \mathrm{~A}$, the DX and D0 magnet currents were kept constant by allowing a crossing angle. This shows a flattening of the $\mathrm{W}$-production rate above $274 \mathrm{GeV}$ when the D0 and DX currents were clamped; however there is still a slow rise in overall rate after the crossing angle starts to open up. Right: Comparison of W-production rates for $15 \pi$ (red) and $20 \pi \mu \mathrm{m}$ (blue) beams.

For the STAR and PHENIX IR's we would still attempt to keep a $0^{\circ}$ crossing angle up to $275 \mathrm{GeV}$. If we were to ramp higher than $278 \mathrm{GeV}$, then the present D0DX cryogenic leads limit the current so that the crossing angle would have to be increased at the STAR and PHENIX IP's (see Fig. 18). Note that for this solution, we have pushed some currents up very close to the limits for some power supplies and leads, so this scenario needs to be examined in detail by the power supply engineers, although our usual constraints were satisfied for fitting the quadrupole currents.

Fig. 18 shows the effect on luminosity and W-production rate vs energy when the DX and D0 currents are clamped at the $274 \mathrm{GeV}$ level. Note that even though the luminosity drops above $274 \mathrm{GeV}$ due to an increasing crossing angle, the production rate still increases with a shallower slope due to the faster rise in cross section. So perhaps a little more rate could be squeezed out if the main dipole supplies were pushed beyond their $5625 \mathrm{~A}$ rating.
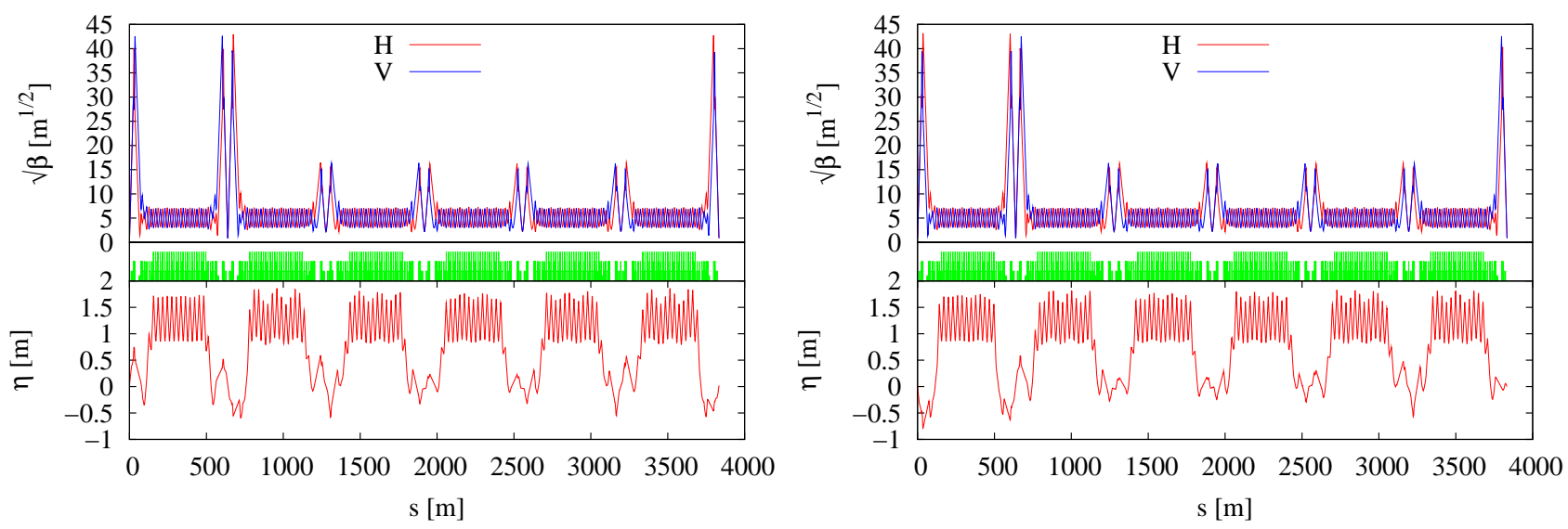

Figure 19. $\sqrt{\beta}$-functions and dispersion $(\eta)$ for a new quadrupole solution at $275 \mathrm{GeV}$ with $\beta^{*}=0.7 \mathrm{~m}$ at STAR and PHENIX. The betatron tunes were kept to the same values as for Fill 10348. The currents in the magnets and cryogenic feedthroughs for quadrupole are within the present constraints for RHIC operation. Left: Blue ring; Right: Yellow ring.

Fig. 19 shows optics functions for a new solution of quadrupole strengths at $275 \mathrm{GeV}$. The currents in the quadrupole magnets (including the 72 trim quadrupoles) and cryogenic feedthroughs are within our present limits for RHIC. We might expect $4 \pm 2$ new training quenches of triplet quadrupoles (QRI) to operate at these currents. The estimate of new training quenches for all the QRG quadrupoles in this scenario is less than one quench. The 
DRG and D0 dipoles are not expected to experience any new training at the $275 \mathrm{GeV}$ level.

Since the DX magnets do not retain the training over thermal cycles, we could expect a similar number of training quenches as previous years for the 8 magnets in IR's other than 6 and 8 . For the higher DX current of $7147 \mathrm{~A}$ at $275 \mathrm{GeV}$ at STAR and PHENIX, we can obtain one estimate by summing the number of original training quenches needed to exceed $7150 \mathrm{~A}$ for the four magnets in Table 2: $5+5+5+3=18$, although the actual number of training quenches may be somewhat lower.

\section{Other considerations not covered in this paper.}

Some other topics which were not discussed in this paper include:

- Depolarizing resonances may be stronger above $250 \mathrm{GeV}$.

- Higher field will be required for the abort kickers.

- There will be an increased radiation load on the beam dumps.

- There is a possibility of more punch-through of spent beam causing beam-induced quenches of quadrupoles downstream of the dump, particularly since they would probably be operating at higher currents.

- Energy dependence of acceptance and efficiencies for the STAR and PHENIX detectors have not been taken into account.

- The effect of different $\beta^{*}$ 's on luminosity were not considered.

- Energy dependence of the nonlinear multipole errors were not considered.

- Any possible upgrades addressing cooling of proton beams during a fill were also not considered.

- At IP 2, a proposed experiment "AnDY" (Drell-Yan measurement of $A_{N}$ ) may require a $0^{\circ}$-crossing angle. The magnet DRX107 installed at g1-dhx in IR 2 has been suspected of having a heat-load problem. If this cannot be repaired, then perhaps the spare DRX114 could be used.

- The electron lenses for beam-beam compensation, to be installed at IR 10, should probably be aligned for a constant horizontal crossing angle (probably $2 \mathrm{mrad}$ ) of the proton beams. Further study of the electron and proton beam trajectories through the various solenoids with proton crossing angles will be required.

\section{Summary}

Increasing the energy of RHIC could double the W-production rate. However the expected number of quenches to reach $325 \mathrm{GeV}$ per beam would be too high ( $\gtrsim 400$ just for arc dipoles).

A more modest goal of $275 \mathrm{GeV}$ would provide almost a $50 \%$ increase in the rate for $\mathrm{W}$-production and seems possible with the present hardware. In this case we might expect up to 18 training quenches for the DX magnets where we allow 2 mrad crossing angles at IR's 24,10 , and 12 and $0^{\circ}$ crossing angles at STAR and PHENIX. We estimate $8 \pm 3$ and $4 \pm 2$ training quenches respectively for the DRG dipoles and QRI triplet quadrupoles.

We do not expect any new training quenches from the eight D0 dipoles around STAR and PHENIX, assuming that they retain their previous training. Even without retention of training, we estimate only about two quenches would be required.

We should note that even though a higher W-production rate may be possible by increasing the energy to $275 \mathrm{GeV}$, the polarization could be lower than at $250 \mathrm{GeV}$, since depolarizing resonance strengths increase with energy. It will be worth while to concentrate on improving the polarization at $250 \mathrm{GeV}$ before embarking on a long run at higher energy.

Since it appears feasible to increase the energy by up to $10 \%$ with the present hardware, it could make sense to try ramping magnets up to these new operating currents without beam at the end of the current Run before shutting down the refrigerator. Since the DX magnets require retraining after every thermal cycle, we suggest that the rings be kept at temperatures below $80 \mathrm{~K}$ after the DX magnets are trained to higher currents.

\section{Acknowledgements}

We would especially like to thank Joe Muratore for providing data files of the initial quench tests for the magnets which were cold tested prior to installation. Animesh Jain analyzed the magnet measurement data to obtain the magnet transfer functions which we then fit to functional forms for this paper. Peter Wanderer and Steve Peggs provided some useful insight on the history of measurements. Don Bruno and Greg Heppner provided information on the retraining of DX magnets after installation in the RHIC tunnel. 
Don Bruno and Anthony DiLieto verified the limits for power supplies and cryogenic feedthroughs, and George Ganetis provided some guidance on the actual limits of the 6300 A feedthroughs. Carl Schultheiss furnished some information on the actual limitations of the present dipole and quadrupole main power supplies Guillaume Robert-Demolaize provided valuable information on the aperture.

Werner Vogelsang calculated the expected increase in W-boson production with higher energy. We would also like to thank Christine Aidala, Mickey Chiu and other members of the PHENIX collaboration for useful

discussions on the production of W-bosons at RHIC. Mike Sivertz and Zhaoming Ma provided useful discussions on error analysis.

\section{References}

$1 \mathrm{~W}$. Vogelsang, private communication.

2 M. Chu, private communication.

3 C. Aidala, private communication.

4 W. W. MacKay, "Luminosity as Calculated from Machine Parameters", C-A/AP/89, BNL, Upton, NY (2002). Also included as Appendix D of M. Conte and W. W. MacKay An Introduction to the Physics of Particle Accelerators, 2nd Ed., World Sci., (2008).

5 W. W. MacKay et al., "Feasibility of Increasing the Energy of RHIC", PAC2001, 3129 (2001).

6 Anonymous, "RHIC Design Manual", Revision Date: October, 2000.

URL: http://www.agsrhichome.bnl.gov/NT-share/rhicdm/00_toc1i.htm

7 S. Tepikian et al., "Tuning Curves" RHIC/AP/23, (1994).

8 S. Peggs, et al., "Interaction Region Closed Orbits", RHIC/AP/135 (1997).

9 Thomas William, Colin Kelley et al., gnuplot, version 4.2, http://www.gnuplot.info/ (2008). 